Aus der Poliklinik für Präventive Zahnmedizin, Parodontologie und Kariologie (Prof. Dr. med. dent. A. Wiegand) im Zentrum Zahn-, Mund- und Kieferheilkunde der Medizinischen Fakultät der Universität Göttingen

\title{
Selbstadhäsive Komposite
}

\section{als Füllungs- und Reparaturmaterialien}

\author{
In-vitro-Studie zur Haftung auf \\ Zahn- und Kompositoberflächen
}

\author{
INAUGURAL-DISSERTATION \\ zur Erlangung des Doktorgrades \\ für Zahnheilkunde \\ der Medizinischen Fakultät der \\ Georg-August-Universität zu Göttingen
}

vorgelegt von

Jana Karen Peterson

aus

Hannover-Langenhagen

Göttingen 2017 
Dekan:

Referent/in

Ko-Referent/in:

Drittreferent/in:
Prof. Dr. rer. nat. H.K. Kroemer

Prof. Dr. med. dent. A. Wiegand

PD Dr. Matthias Rödiger

Prof. Dr. mult. Thomas Meyer

Datum der mündlichen Prüfung: 22.03.2018 
Hiermit erkläre ich, die Dissertation mit dem Titel „Selbstadhäsive Komposite als Füllungs- und Reparaturmaterialien In-vitro-Studie zur Haftung auf Zahn- und Kompositoberflächen“ eigenständig angefertigt und keine anderen als die von mir angegebenen Quellen und Hilfsmittel verwendet zu haben.

Göttingen, den 04.10.2017 


\section{Inhalt}

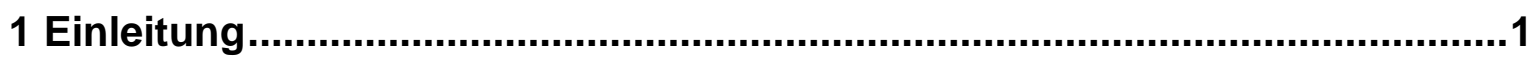

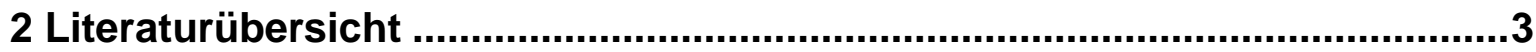

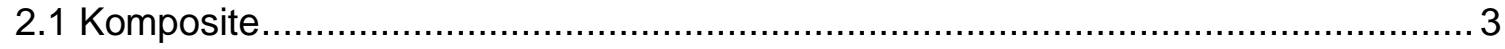

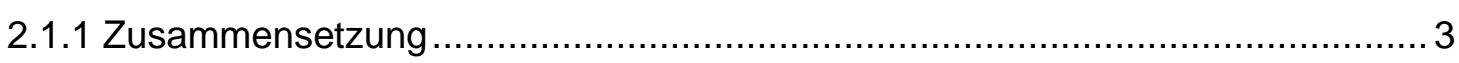

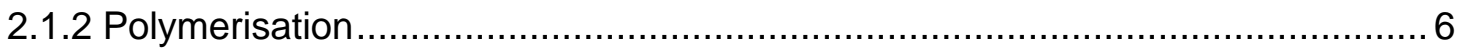

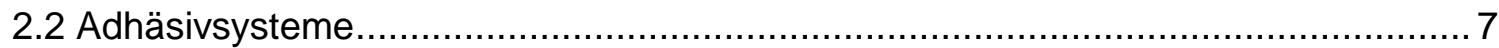

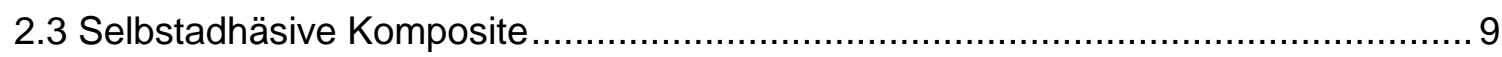

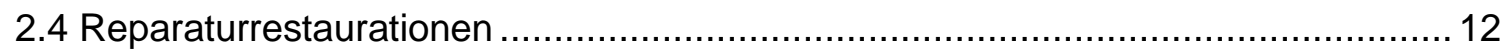

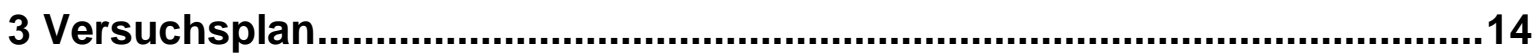

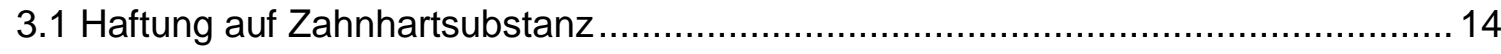

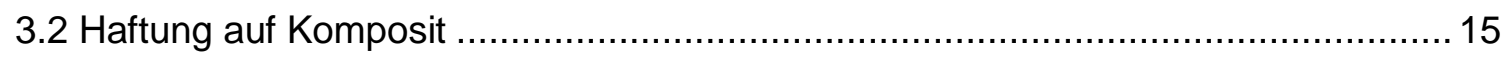

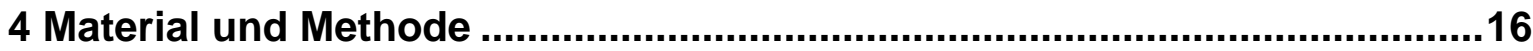

4.1 Herstellung der Prüfkörper - Haftung auf Zahnhartsubstanz .................................. 16

4.2 Herstellung der Prüfkörper - Haftung auf Komposit ............................................. 19

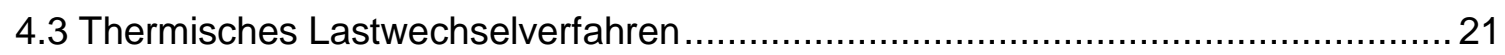

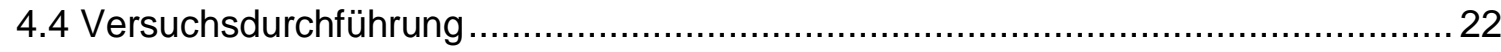

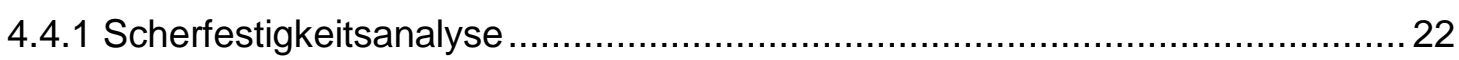

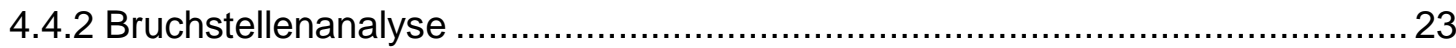

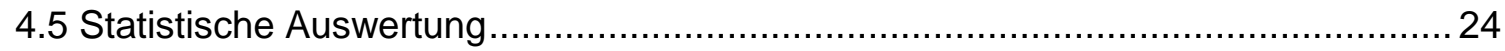

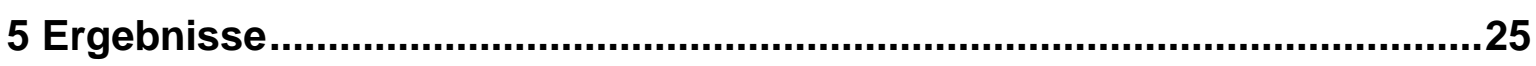

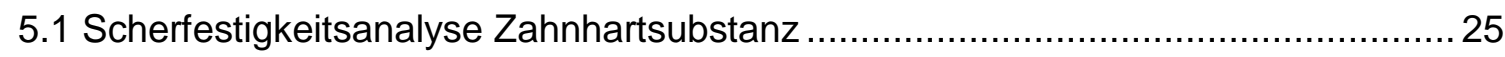

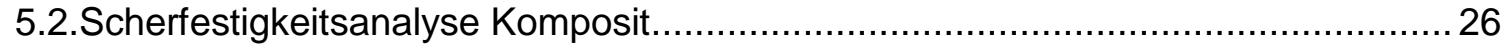

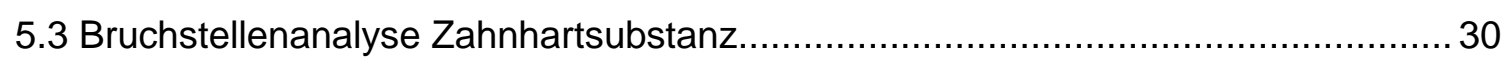

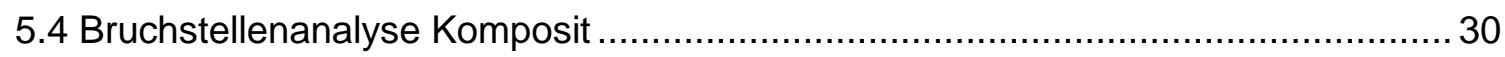

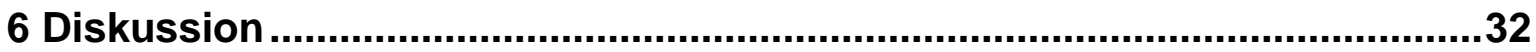

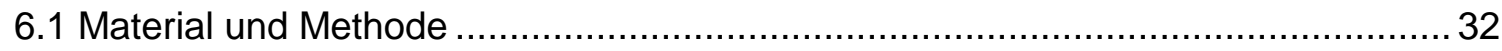

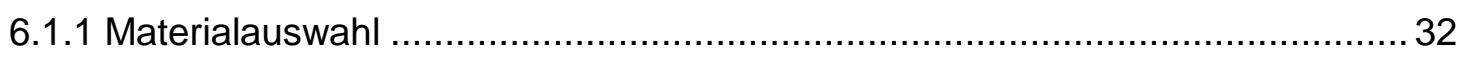


6.1.2 Simulation der Kompositreparatur ........................................................ 33

6.1.3 Thermisches Lastwechselverfahren ........................................................ 34

6.1.4 Scherfestigkeits- und Bruchstellenanalyse ............................................... 36

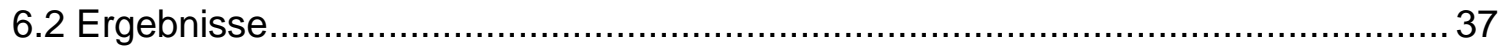

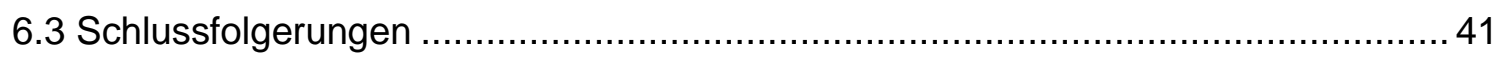

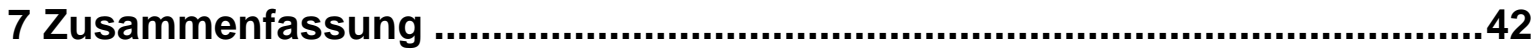

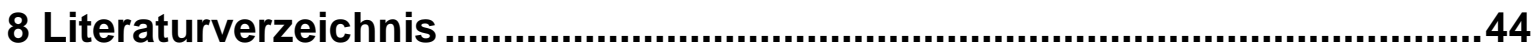




\section{Einleitung}

Komposite sind in der Zahnmedizin schon seit langem als Materialien für zahnfarbene direkte Restaurationen etabliert. Ihre Applikation gestaltet sich jedoch als recht techniksensibel und zeitaufwendig, da die Zahnoberfläche zuvor mit einem Adhäsivsystem konditioniert werden muss (Van Meerbeek et al. 2003; Van Meerbeek et al. 2005; Spreafico et al. 2006; Magne et al. 2008). Das Adhäsivsystem ermöglicht eine stabile Verbindung zwischen hydrophobem Komposit und hydrophilem Dentin, indem es in freigelegtes Kollagen penetriert und dort eine Hybridschicht zwischen beiden Materialien ausbildet (Van Meerbeek et al. 2003).

Um die Kompositapplikation zu simplifizieren, wurde zunächst die Anwendung der Adhäsivsysteme vereinfacht. Durch die Zusammenfassung verschiedener Anwendungsschritte und Komponenten existieren heute neben den klassischen, mehrschrittig anzuwendenden Adhäsivsystemen auch Systeme, bei denen nur ein einziger Applikationsschritt durchzuführen ist.

Als weitere Konsequenz wurde die Entwicklung selbstadhäsiver Komposite in Erwägung gezogen, um vollumfänglich auf die separate Konditionierung der Zahnhartsubstanz mit einem Adhäsivsystem verzichten zu können. Nachdem vor einigen Jahren zunächst selbstadhäsive Kompositzemente für die Befestigung indirekter Restaurationen oder Stiftsysteme etabliert worden waren, entwickelten verschiedene Hersteller selbstadhäsive Komposite für die Herstellung direkter Restaurationen, deren Anwendung ohne eine vorherige Konditionierung der Zahnoberfläche mit einem Adhäsivsystem möglich sein soll. Die Funktionsweise dieser Komposite basiert auf ihren selbstätzenden und/oder selbstadhäsiven Monomeren, die die Schmelz- und Dentinoberflächen anätzen bzw. chemisch an das Hydroxylapatit der Zahnhartsubstanz binden sollen (Poitevin et al. 2013).

Während schon recht viele Untersuchungen zu selbstadhäsiven Befestigungskompositen durchgeführt wurden, liegen kaum Daten zu selbstadhäsiven Kompositen als direkte Füllungswerkstoffe vor, obwohl diese schon seit einigen Jahren auf dem Markt erhältlich sind.

Die vorliegenden Studien zeigen für die selbstadhäsiven Komposite bereits unmittelbar nach ihrer Applikation geringere Haftwerte als für konventionelle Komposite, die mit Adhäsivsystemen in der Etch\&Rinse- oder Self-Etch-Technik 
verwendet wurden (Juloski et al. 2012; Goracci et al. 2013; Veli et al. 2014; Makishi et al. 2015; DMG America). Bislang gibt es kaum Informationen darüber, wie sich Alterungsprozesse und Degradation in der Mundhöhle auf die Haftung der selbstadhäsiven Komposite auswirken.

Weiterhin ist nicht bekannt, ob sich selbstadhäsive Komposite möglicherweise auch als Reparaturmaterialien für defekte Restaurationen eignen. Die Reparatur dentaler Restaurationen ist zahnhartsubstanzschonender und möglicherweise kosteneffektiver als die komplette Erneuerung einer nur partiell insuffizienten Restauration (Moncada et al. 2009; Fernández et al. 2015; Kanzow et al. 2016b) und hat daher im Sinne einer minimalinvasiven Zahnheilkunde breite Akzeptanz gewonnen (Kanzow et al. 2016a).

Für eine erfolgreiche Reparatur von defekten Restaurationen ist jedoch ebenfalls eine umfangreiche Konditionierung der Reparaturoberfläche (z. B. Anrauen oder Silikatisierung, Verwendung von Silan-Lösungen und/oder Adhäsivsystemen) Voraussetzung. Möglicherweise könnte der Einsatz von selbstadhäsiven Kompositen diesen Vorgang weniger aufwendig und techniksensibel gestalten.

Das Ziel dieser Studie war es daher, selbstadhäsive fließfähige Komposite als direkte Füllungsmaterialien sowie ihren Einsatz als Reparaturmaterialien für Kompositrestaurationen zu prüfen. Dafür sollte die Scherfestigkeit auf Schmelzund Dentin- sowie auf Nano-Hybridkompositprüfkörpern untersucht werden.

Als Nullhypothese wurde definiert, dass die Haftwerte der selbstadhäsiven fließfähigen Komposite sich nicht signifikant von einem konventionellen fließfähigen Komposit unterscheiden. 


\section{Literaturübersicht}

\subsection{Komposite}

\subsubsection{Zusammensetzung}

Komposite werden in der Zahnmedizin schon seit langem als zahnfarbene Füllungswerkstoffe angewendet. Als plastische Materialien erhärten sie durch Energie-induzierte Polymerisation (Cramer et al. 2011). Dentale Komposite setzen sich aus drei Hauptbestandteilen zusammen: organische Matrix, disperse Phase und Verbundphase.

Hauptbestandteil der organischen Matrix im Grundzustand sind die KompositMonomere. Es handelt sich meist um mehrfunktionelle Methacrylate, die aus einem organischen Zwischenglied zwischen zwei Methacrylsäureester-Resten bestehen. Das organische Zwischenglied ist ausschlaggebend für die mechanischen und physikalischen Eigenschaften des Komposits, während über die Methacrylsäureester-Reste einzelne Monomere verknüpft und somit langkettige und vernetzte Komposit-Polymere gebildet werden können. Häufig eingesetzte Monomere (Abb. 2.1) sind unter anderem Bis-GMA (Bisphenol-Amethacrylat), EGDMA (Ethylenglykoldimethacrylat), TEGDMA (Triethylenglycoldimethacrylat) und UDMA (Urethandimethacrylat) (Peutzfeldt 1997; Ferracane 2011; Ilie und Hickel 2011).

Enthalten die Komposit-Monomere sehr viele Sauerstoffatome (-O) oder Hydroxylgruppen (-OH), ist die Wasseraufnahme der Matrix erhöht. Es kommt zu einer hygroskopischen Expansion des Komposits, die mechanischen Eigenschaften und die Verschleißrate verschlechtern sich (Wei et al. 2013).

Auch die Molekülkettenlänge der Monomere nimmt Einfluss auf die Kompositeigenschaften. Je kurzkettiger ein Monomer, desto größer ist die Schrumpfung bei der Polymerisation. Durch die so entstehenden Spannungen innerhalb des Materials werden die mechanischen Eigenschaften verschlechtert. Längere Molekülketten führen dagegen zu einer erhöhten Viskosität. Hochvisköse Komposite können Oberflächen schlechter benetzen und lassen sich schwieriger verarbeiten. Um möglichst gute Kompositeigenschaften (eine geringe Polymerisationsschrumpfung bei herabgesetzter Viskosität) zu erreichen, werden in dentalen Kompositen oft langkettige Monomere mit kurzkettigen Komonomeren 
(z. B. TEGDMA, EGDMA) gemischt (Janda 1988).<smiles>C=C(C)C(=O)OCC(O)COc1ccc(C(C)(C)c2ccc(OCC(O)COC(=O)C(=C)C)cc2)cc1</smiles>

Bis-GMA<smiles>C=C(C)C(=O)OCCOC(=O)C(=C)C</smiles>

EGDMA<smiles>C=C(C)C(=O)OCCOCCOCCOC(=O)C(=C)C</smiles>

TEGDMA<smiles>C=C(C)C(=O)OCCOC(=O)NCC(C)(C)CC(C)CCNNC(=O)OCCOC(=O)C(=C)C</smiles>

UDMA

Abbildung 2.1: Chemische Strukturformeln typischer Kompositmonomere

Die disperse Phase besteht aus anorganischen Füllkörpern und dient dazu, die physikalischen und chemischen Eigenschaften der Kompositmatrix zu verbessern. Sie erhöht die Druck- und Biegefestigkeit sowie die Verschleißfestigkeit und beeinflusst das E-Modul positiv. Die Polymerisationsschrumpfung und Wasseraufnahme werden gesenkt (Janda 1988; Manhart et al. 2001). 
In zahnmedizinischen Kompositen werden hauptsächlich Keramik, Quarz und Siliziumdioxid als Füllstoffe verwendet (Peutzfeldt 1997).

Sowohl der Füllstoffgehalt als auch die Form und Größe der Füllkörper beeinflussen das klinische Verhalten und die Eigenschaften von Kompositen, wie z. B. ihre Verarbeitbarkeit, ihr Abrasionsverhalten und ihre Polierbarkeit sowie ästhetischen Eigenschaften (Lutz und Phillips 1983; llie und Hickel 2011).

Durch Verringerung des Füllstoffanteils oder durch Zusatz von verdünnenden Matrixbestandteilen (z. B. TEGDMA, s.o.) entstehen fließfähige Komposite. Sie weisen zwar schlechtere mechanische Festigkeitswerte und eine höhere Polymerisationsschrumpfung auf, haben aber den Vorteil, durch die erniedrigte Viskosität eine besonders gute Oberflächenbenetzung zu erreichen (Cramer et al. 2011; Baroudi und Rodrigues 2015).

Die Klassifikation dentaler Komposite erfolgt heute nach Art und Größe ihrer Füllkörper. Die Füllkörpergrößen varieren dabei zwischen $0,005 \mu \mathrm{m}$ bei Nanofüllern und ca. $50 \mu \mathrm{m}$ bei Makrofüllern (Ferracane 2011). So enthalten z. B. Nano-Hybridkomposite 80-85 Gew.-\% Nanofüllkörper und zeichnen sich durch gute mechanische und physikalische Eigenschaften kombiniert mit einer ansprechenden Ästhetik aus (Ilie und Hickel 2011; Patel et al. 2016).

Die Verbundphase dient der Einbettung der anorganischen Füllkörper in die organische Kompositmatrix. Die Füllkörper haften entweder mechanisch oder durch chemische Verbindung in der Matrix (Abb. 2.2). Um eine chemische Verbindung zu erreichen, werden die Füllkörper durch Silanisierungsmittel hydrophobiert (Janda 1988; Cramer et al. 2011). Die Verbundphase kann jedoch durch saure Hydrolyse in der Mundhöhle desintegriert werden, es kommt zum Füllkörperverlust. Die Silanisierung ist daher als eine Schwachstelle anzusehen (Lutz und Phillips 1983). 


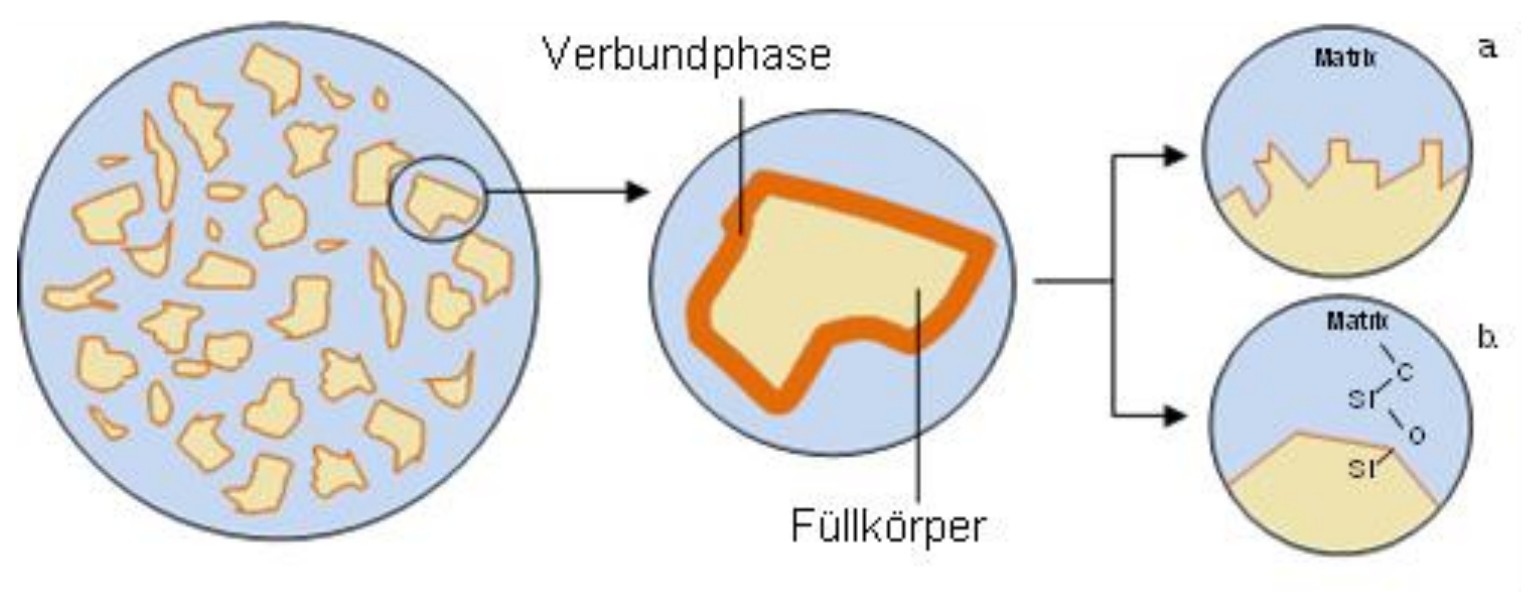

Abbildung 2.2: Kompositaufbau: Die Füllkörper sind entweder mechanisch (a.) oder chemisch (b.) durch die Verbundphase in der Kompositmatrix verankert.

\subsubsection{Polymerisation}

Bei der Kompositpolymerisation handelt es sich um eine Energie-induzierte, radikalische Polymerisation. Die einzelnen Methacrylatgruppen werden zu einer dreidimensional vernetzten Matrix verknüpft (Cramer et al. 2011).

Dabei zerfallen Initiatormoleküle zu sehr reaktiven Radikalen und öffnen die Doppelbindungen der Methacrylate. So entstehen Methacrylat-Radikale, die durch ihre Aktivierung eine Kettenreaktion auslösen und an weitere Methacrylate binden, die dadurch ebenfalls zu Radikalen umgeformt werden und so weiter reagieren können (Cramer et al. 2011).

Der Polymerisationsgrad lichthärtender Komposite ist neben der Intensität und Wellenlänge der Lichtquelle auch vom Abstand der Lichtquelle, der Bestrahlungsstärke und -dauer sowie der Zusammensetzung und Farbe des Komposits abhängig. Konventionelle Komposite lassen sich nur bis zu einer Schichtstärke von maximal $2 \mathrm{~mm}$ suffizient aushärten.

Durch die Vernetzungsreaktion der Monomere und der damit verbundenen Änderung der molekularen Strukturen kommt es während der Polymerisation zu einer Schrumpfung des Materials. Es entstehen Spannungen, die zu Rissen und Randspaltbildungen führen können und die Materialeigenschaften verschlechtern. Um die bei der Applikation entstehenden Spannungen möglichst gering zu halten, hat es sich bewährt, das Komposit schichtweise in Inkrementen in die Kavität zu applizieren (Ferracane 2008). 


\subsection{Adhäsivsysteme}

Komposite allein erreichen keine ausreichende Haftung auf Zahnhartsubstanzen (Manhart et al. 2004; Garcia-Godoy et al. 2010). Aufgrund der fehlenden Konformität von hydrophilem Dentin und hydrophobem Komposit sowie der Kompositschrumpfung während der Polymerisation ist eine stabile Verbindung zwischen Zahnhartsubstanz und Komposit ohne die Vorbehandlung mit einem Adhäsivsystem nicht möglich (Garcia-Godoy et al. 2010; Peumans et al. 2012; Poitevin et al. 2013).

Daher macht die Applikation konventioneller Komposite die vorhergehende Konditionierung der Zahnoberfläche mit einem Adhäsivsystem obligat.

Adhäsivsysteme basieren auf mehreren Komponenten (Säure, Primer, Bonding), die Mikroretentionen in der Zahnoberfläche schaffen und diese aufgrund ihrer niedrigen Viskosität besonders suffizient benetzen können und so über sogenannte Micro-Tags (Kunststoffzapfen) eine mikromechanische Haftung an der Zahnoberfläche ermöglichen (Van Meerbeek et al. 2003).

Als härteste Struktur des menschlichen Körpers besteht der Zahnschmelz zu ca. 96-97 Gew.-\% aus anorganischen Anteilen, der Rest setzt sich hauptsächlich aus organischen Bestandteilen wie Proteinen und Wasser zusammen. Bei der anorganischen Substanz handelt es sich um Hydroxylapatitkristalle, die aus Kalziumphosphatverbindungen bestehen und durch ihre parallele Ausrichtung sogenannte Schmelzprismen bilden (Mjør und Fejerskov 1986).

Dentin ist im Gegensatz zum Schmelz ein lebendes Gewebe. Sein anorganischer Anteil beträgt nur ca. 70 Gew.-\%, der Rest besteht aus organischem Material und Wasser (Mjør und Fejerskov 1986). Der anorganische Teil setzt sich, wie auch beim Schmelz, aus Hydroxylapatitkristallen zusammen, der organische Anteil besteht hauptsächlich aus Kollagen. Das Dentin wird von Dentinkanälchen, den Tubuli, durchzogen.

Die Haftung von Kompositen an Zahnschmelz ist verhältnismäßig einfach zu erreichen. Durch Säureätzung entsteht auf Schmelz ein retentives Ätzmuster entlang der Schmelzprismen, das die mikromechanische Verankerung von Kompositen begünstigt (Van Meerbeek et al. 2003; Frankenberger und Tay 2005; Garcia-Godoy et al. 2010). Als ideal hat sich hierbei die Anwendung von 37\%iger Phosphorsäure für $30 \mathrm{~s}$ erwiesen (Gwinnett 1981; Van Meerbeek et al. 2003). 
Der Verbund zwischen Komposit und Zahnschmelz wird schließlich durch funktionelle Monomere eines niedrigviskösen Kunststoffs (sog. Bonding) erreicht, die in die durch den Ätzvorgang geschaffenen Freiräume diffundieren können und so für eine mikromechanische Verankerung sorgen (Van Meerbeek et al. 2003; Hickel et al. 2004).

Die Haftung eines hydrophoben Komposits an dem hydrophilen Dentin ist wesentlich schwieriger zu erreichen. Hinzu kommt, dass durch mechanische Bearbeitung an der Dentinoberfläche eine Schmierschicht entsteht, die die Konditionierung weiter erschwert (Van Meerbeek et al. 1992; Oliveira et al. 2003).

Auch das Dentin wird zunächst mit Säuren geätzt, wobei entweder 37\%ige Phosphorsäure oder säurehaltige „Primer" verwendet werden können. Durch die Säureätzung wird das Dentin entlang der Tubuli oberflächlich demineralisiert und das Kollagen freigelegt, die Schmierschicht wird entfernt (Perdigao et al. 1996; Perdigao et al. 2000; Perdigao und Frankenberger 2001). Amphiphile Monomere des „Primers“ können in die demineralisierte Oberfläche eindringen und erlauben außerdem eine Benetzung mit dem vergleichsweise hydrophoben „Bonding“, das wiederum die Haftung des Komposites ermöglicht. Aus freigelegtem Kollagen, "Primer" und "Bonding“ entsteht eine durch Polymerisation stabilisierte Hybridschicht. Das freigelegte Kollagennetzwerk im Dentin muss vollständig von Monomeren des Adhäsivsystems benetzt werden, da es sonst zu einer Schwächung des Verbundes kommen kann (Frankenberger et al. 2000).

Lange galt sowohl auf Schmelz als auch auf Dentin die Etch\&Rinse-Technik als effektivste Adhäsivtechnik (Van Meerbeek et al. 2003). Dabei werden zunächst Zahnschmelz und Dentin simultan mit Phosphorsäure geätzt und diese dann abgespült, anschließend wird ein "Primer" in die Zahnoberflächen einmassiert und ein „Bonding“ aufgebracht und polymerisiert.

Da sich die Anwendung von Etch\&Rinse-Systemen als recht kompliziert und zeitaufwendig gestaltet, wurden zunächst sog. Self-Etch-Adhäsive entwickelt, die saure Monomere enthalten und den separaten Schritt der Phosphorsäureapplikation unnötig machen. Als weitere Vereinfachung wurden Adhäsivsysteme entwickelt, bei denen mehrere Komponenten in einem Anwendungsschritt zusammengefasst sind (sog. All-in-one- bzw. One-bottleAdhäsivsysteme). 
Während die Haftwerte der All-in-one-Systeme auf Zahnschmelz nicht an die der Etch\&Rinse-Systeme heranreichen (Van Meerbeek et al. 2003), wird auf Dentin bei der Verwendung milder Self-Etch-Adhäsive sogar eine Steigerung der Haftwerte beobachtet (Ozer und Blatz 2013).

Milde Self-Etch-Adhäsive weisen einen pH-Wert von ca. 2 auf und lösen bei ihrer Anwendung die Hydroxylapatitkristalle nur leicht an, sodass sie als Schutz für das Kollagengeflecht im Dentin erhalten bleiben. Weiterhin enthalten sie meist funktionelle Monomere, die eine chemische Verbindung mit dem Kalzium des Hydroxylapatits im Zahn eingehen. Aufgrund ihrer nur milden Ätzwirkung sind diese Self-Etch-Adhäsive nicht in der Lage, den Schmelz tief genug zu demineralisieren, um eine mikromechanische Verankerung zu schaffen. So erklären sich möglicherweise die niedrigen Haftwerte auf Schmelz.

\subsection{Selbstadhäsive Komposite}

Vor der Entwicklung selbstadhäsiver Komposite als Materialien für die direkte Füllungstherapie wurden selbstadhäsive Kompositzemente für die Befestigung indirekter Restaurationen und Stiftsysteme schon seit mehreren Jahren eingesetzt. Vor allem bei der Befestigung keramischer Inlays und Teilkronen kann durch die Verwendung von Kompositzementen Zahnhartsubstanz geschont und stabilisiert werden (Mehl et al. 2004). Klassische Befestigungskomposite setzen jedoch - ebenso wie Komposite für die direkte Füllungstherapie - eine Konditionierung der Zahnhartsubstanz sowie des keramischen Werkstückes mit einem Adhäsivsystem voraus, sodass das Einsetzen indirekter Restaurationen durch die Verwendung selbstadhäsiver Kompositzemente simplifiziert werden konnte.

Selbstadhäsive Kompositzemente sind Hybridmaterialien, die Eigenschaften von Kompositen, selbstätzenden Adhäsiven und dentalen Zementen in sich vereinen und den zusätzlichen Einsatz eines Adhäsivsystems überflüssig machen. Eine Verbindung zur Zahnhartsubstanz wird über Methacrylat-Monomere mit Phosphorsäure- oder Carboxylgruppen erreicht, die Schmelz und Dentin demineralisieren bzw. mit dem Kalzium der Zahnhartsubstanz Salze bilden und so chemisch an die Zahnhartsubstanz binden (Ferracane et al. 2011). Selbstadhäsive 
Kompositzemente zeigen jedoch eine geringere Abrasionsstabilität und eine vermehrte Degradationsanfälligkeit als Komposite für direkte Restaurationen, was unter anderem auf das Fehlen einer Silanisierung der Füllkörper zurückgeführt wird (Belli et al. 2009; Ferracane et al. 2011). Daher war ihr Einsatz zunächst auf die Anwendung als Befestigungsmaterialien für indirekte Restaurationen beschränkt.

Schließlich gelang es jedoch verschiedenen Herstellern, auch selbstadhäsive Komposite für die direkte Füllungstechnik zu entwickeln, die eine vorherige Konditionierung der Zahnhartsubstanz mit einem Adhäsivsystem überflüssig machen. Selbstadhäsive Komposite sollen zunächst in einer dünnen Schicht aktiv eingebürstet werden, um die Interaktion ihrer funktionellen Monomere mit der Zahnoberfläche zu verstärken. Nach Lichtpolymerisation der dünnen initialen Schicht können weitere Schichten appliziert werden.

Die funktionellen Monomere agieren selbstätzend und/oder selbstadhäsiv und sind in der Lage Schmelz- und Dentinoberflächen zu konditionieren bzw. chemisch an Hydroxylapatit zu binden (Poitevin et al. 2013).

Als funktionelle Monomere werden z. B. 4-META (4-Methacryloxyethyl-trimelliticsäure), GPDM (Glycerophsophat-Dimethacrylat) oder MDP (10-Methacryloyloxydecyldihydrogenphosphat) verwendet (Abb. 2.3). 


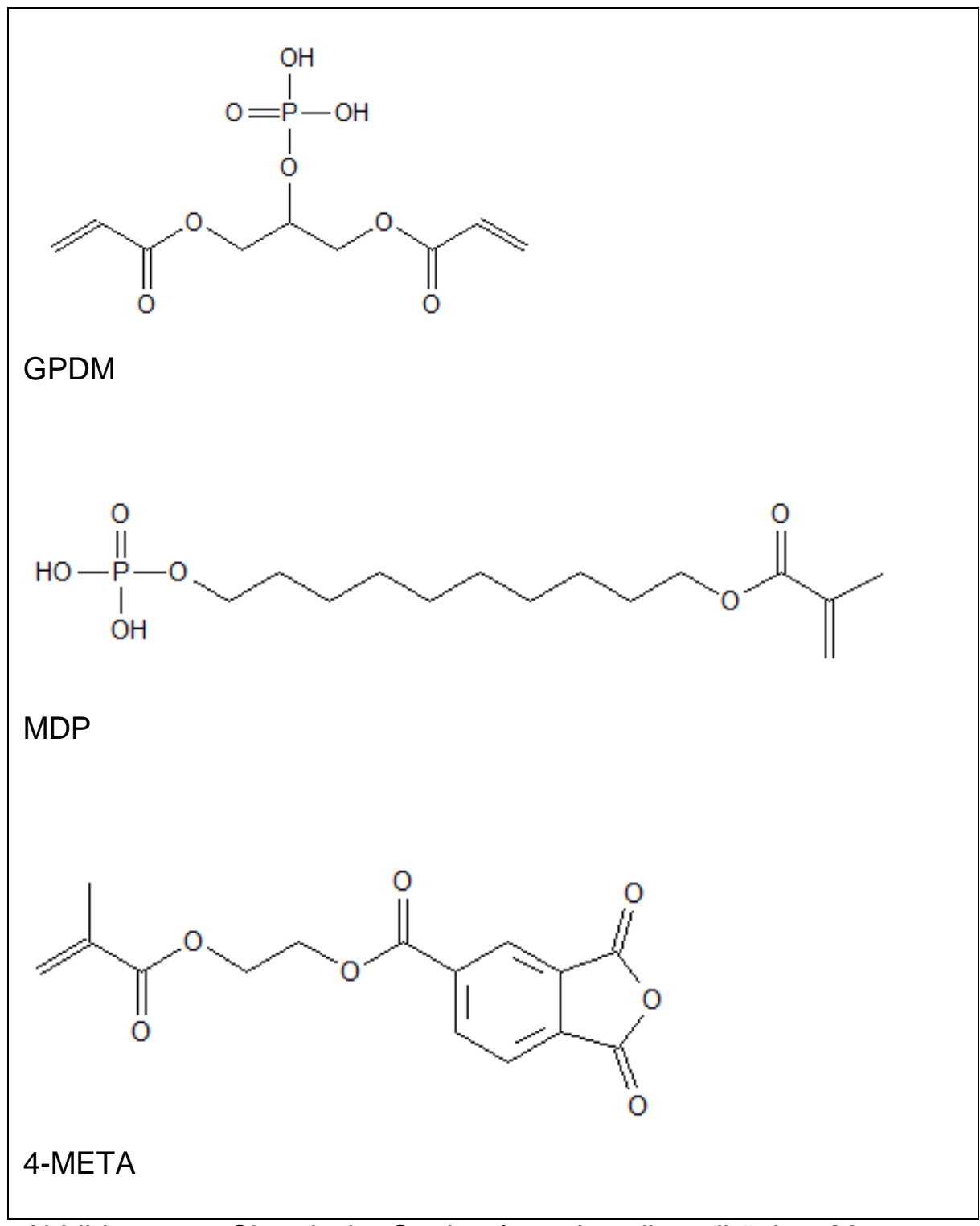

Abbildung 2.3: Chemische Strukturformeln selbstadhäsiver Monomere

Anders als bei der Applikation konventioneller Komposite, bei denen durch die vorhergehende Säure-Ätz-Technik und Anwendung eines Adhäsivsystems eine mikromechanische Haftung an der Zahnhartsubstanz erreicht wird, soll die Haftwirkung der selbstadhäsiven Komposite hauptsächlich auf chemischen Bindungen beruhen. Dabei handelt es sich um lonenbindungen, bei denen die funktionellen Gruppen der selbstadhäsiven Monomere mit den Kalziumionen des Hydroxylapatits aus der Zahnhartsubstanz Salze bilden. 
Mechanische Festigkeit erreichen selbstadhäsive Komposite durch die Vernetzung ihrer Methacrylatgruppen, die nach demselben Prinzip wie bei konventionellen Kompositen abläuft.

Obwohl sie für die klinische Verwendung zugelassen sind, zeigen selbstadhäsive Komposite in vorliegenden Studien direkt nach ihrer Applikation schlechtere Haftwerte als konventionelle Komposite, die mit Adhäsivsystemen angewendet wurden (Juloski et al. 2012; DMG America; Goracci et al. 2013; Veli et al. 2014; Makishi et al. 2015; Brueckner et al. 2017). Es fehlen weiterhin Informationen, inwiefern Alterungsprozesse und Degradation in der Mundhöhle Einfluss auf die Eigenschaften selbstadhäsiver Komposite nehmen.

\subsection{Reparaturrestaurationen}

Wie bei allen zahnärztlichen Restaurationen ist die Lebensdauer direkter Kompositfüllungen mit einer mittleren jährlichen Verlustrate von 2,2\% limitiert. Als häufigster Grund für eine Erneuerung von Kompositrestaurationen werden Sekundärkaries und Füllungsfrakturen genannt (Manhart et al. 2004).

Bei nur partiell insuffizienten Restaurationen kann der Zeitpunkt der Erneuerung der kompletten Restauration zunächst durch eine Reparatur nach hinten verschoben werden. Reparaturrestaurationen haben im Sinne einer minimalinvasiven Zahnheilkunde an Bedeutung und Akzeptanz gewonnen (Kanzow et al. 2016a), ist diese Verfahrensweise doch Zahnhartsubstanz-schonender und kosteneffektiver als die komplette Erneuerung einer nur partiell insuffizienten Restauration (Moncada et al. 2009; Fernández et al. 2015; Kanzow et al. 2016b). In der Regel wird zur Reparatur von defekten Restaurationen Komposit verwendet. In Abhängigkeit von dem Restaurationsmaterial der Originalrestauration sind verschiedene Konditionierungsmaßnahmen notwendig, um eine Haftung des Komposits an der Reparaturoberfläche zu ermöglichen. Diese Konditionierungsmaßnahmen schließen sowohl eine mechanische Bearbeitung (Silikatisierung, Sandstrahlen oder Anrauen mit Diamantschleifer) als auch eine chemische Konditionierung (Silan-Lösung und/oder Adhäsivsystem) ein.

Eine mechanische Bearbeitung der Reparaturoberfläche schafft zum einen Unregelmäßigkeiten und somit eine Vergrößerung der Oberfläche, zum anderen 
entfernt sie die oberste gealterte Kompositschicht und legt darunter eine saubere Kompositfläche frei, die noch nicht direkt mit Speichel und anderen Mundhöhlenbestandteilen kontaminiert wurde (Hannig et al. 2006). Beim Silikatisieren werden zudem Silikatpartikel in die Reparaturoberfläche eingelagert (Rodrigues Jr. et al. 2009), die die Anhaftung des Reparaturkomposits verbessern sollen.

Die zusätzliche Anwendung einer Silan-Lösung führt nicht nur zu einer verbesserten Benetzbarkeit, sie beeinflusst auch die chemische Bindung an anorganische Füllerpartikel oder die Kompositmatrix (Rodrigues Jr. et al. 2009; Maneenut et al. 2011). Adhäsivsysteme sind aufgrund ihrer niedrigen Viskosität in der Lage Mikrorauigkeiten der Reparaturoberfläche besser zu benetzen als die meist visköseren Komposite und sorgen so für zusätzlich verbesserte Haftwerte (Loomans et al. 2011).

Die Anwendung selbstadhäsiver Komposite als Reparaturmaterialien ist zum Zeitpunkt der Studiendurchführung noch nicht untersucht worden. Möglicherweise könnte durch ihre Anwendung das Protokoll für die Durchführung von Kompositreparaturen vereinfacht werden. 


\section{Versuchsplan}

\subsection{Haftung auf Zahnhartsubstanz}

Es werden 64 Schmelz- und 64 Dentinprüfkörper hergestellt und in vier Gruppen (je $n=16$ ) aufgeteilt. Es wird entweder ein selbstadhäsives fließfähiges Komposit oder ein konventionelles fließfähiges Komposit nach Konditionierung mit einem Etch\&Rinse-Adhäsivsystem appliziert.

Nach artifizieller Alterung im Thermolastwechsel wird die Scherfestigkeit gemessen und eine mikroskopische Bruchstellenanalyse durchgeführt (Abb. 3.1).

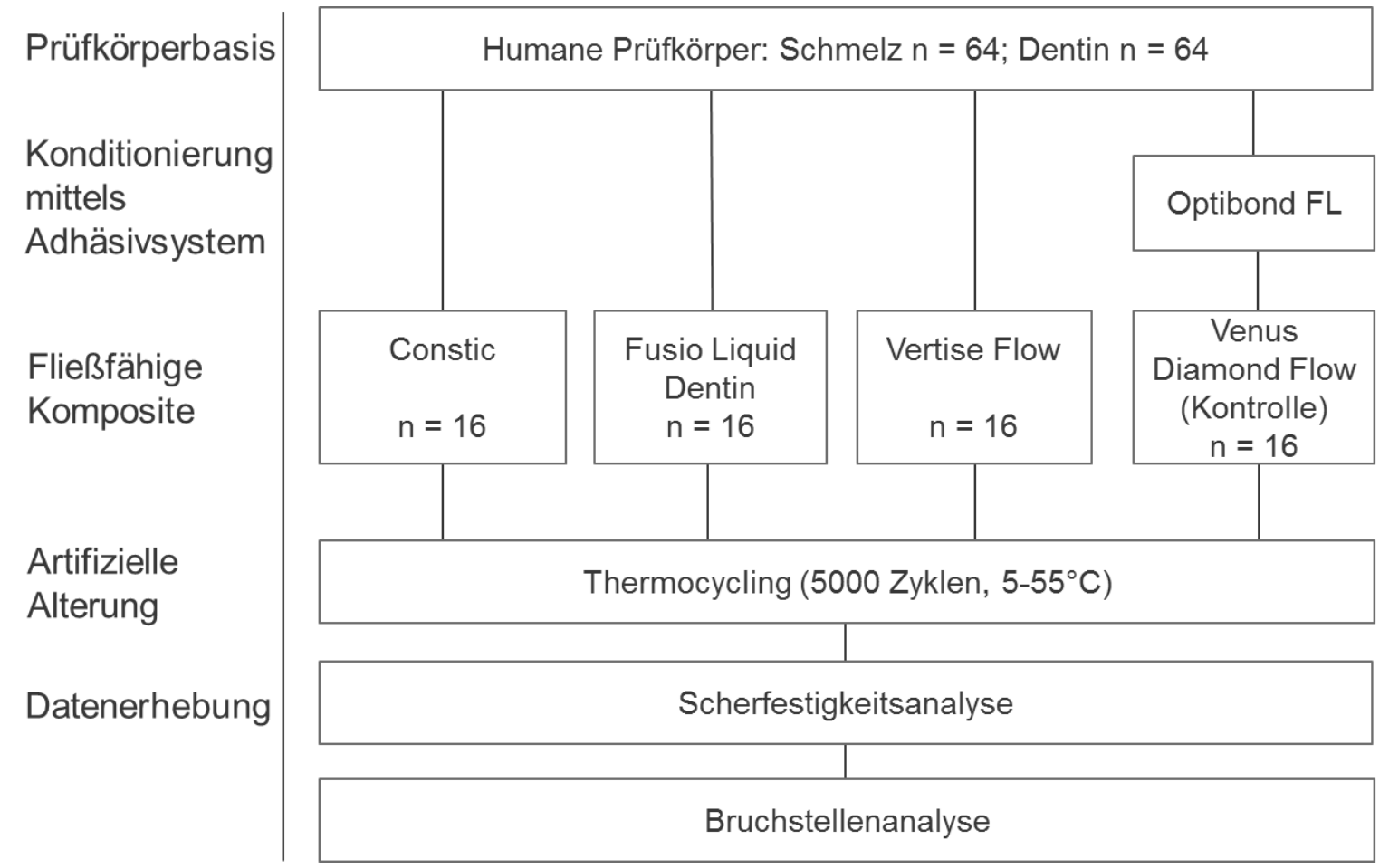

Abbildung 3.1: Versuchsplan zur Haftung auf Zahnhartsubstanz 


\subsection{Haftung auf Komposit}

Es werden 512 Prüfkörper aus einem Nano-Hybrid Komposit hergestellt und im Thermolastwechsel artifiziell gealtert. Um eine Füllungsreparatur zu simulieren erfolgt zunächst eine zufällige Aufteilung in vier Gruppen $(n=128)$, die jeweils einer unterschiedlichen Oberflächenbearbeitung unterzogen (mechanisches Anrauen, Sandstrahlen mit $\mathrm{Al}_{2} \mathrm{O}_{3}$, Silikatisierung und Silanisierung) oder unbehandelt belassen werden.

Die Hälfte der Prüfkörper in jeder Gruppe $(n=64)$ wird nun mittels eines Adhäsivsystems konditioniert. Anschließend wird ein selbstadhäsives fließfähiges Komposit bzw. ein konventionelles fließfähiges Komposit appliziert.

Die Versuchsgruppen $(n=16)$ unterscheiden sich folglich in der Art der Oberflächenbearbeitung, der Konditionierung sowie des eingesetzten fließfähigen Komposits.

Sämtliche Versuchsgruppen werden nun erneut im Thermolastwechsel artifiziell gealtert. Dann wird in einer Universal-Prüfmaschine die Scherfestigkeit gemessen sowie eine mikroskopische Bruchstellenanalyse durchgeführt (Abb. 3.2).

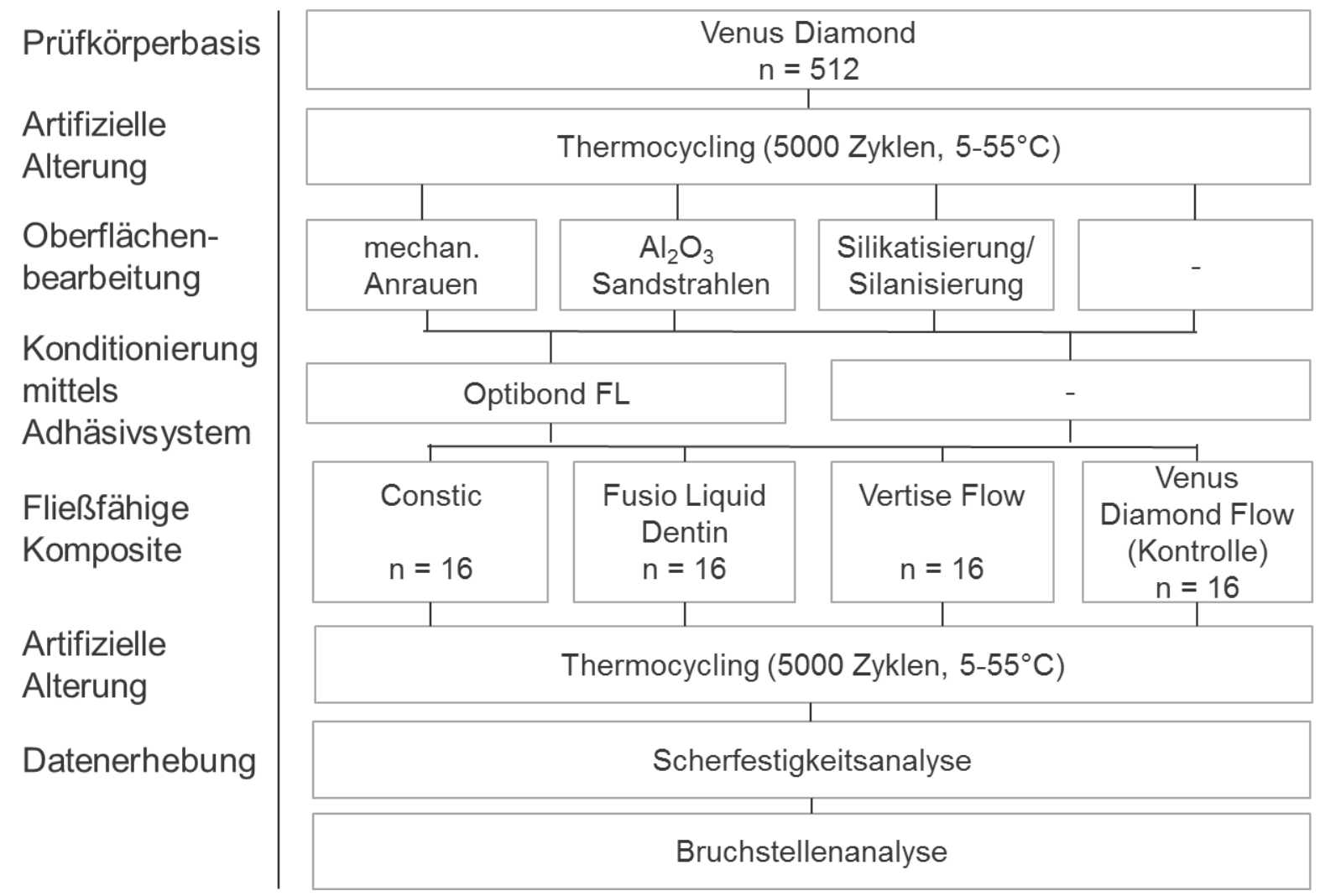

Abbildung 3.2: Versuchsplan zur Haftung auf Komposit 


\section{Material und Methode}

In dieser Studie wurden die in Tabelle 4.1 dargestellten selbstadhäsiven fließfähigen Komposite sowie ein konventionelles fließfähiges Komposit verwendet.

\subsection{Herstellung der Prüfkörper - Haftung auf Zahnhartsubstanz}

Es wurden insgesamt $n=64$ Schmelz- und $n=64$ Dentinprüfkörper hergestellt. Dafür wurden extrahierte und nicht kariöse humane permanente Molaren entsprechend dem Ethikvotum Nr. 27/8/13 verwendet. Mit einer wassergekühlten Säge (Exakt 300 Diamant-Bandsäge, Walter Messner $\mathrm{GmbH}$ ) wurden die Zahnkronen von den Wurzeln getrennt. Die Kronen wurden dann in Kunststoff eingebettet und bis auf den Schmelz bzw. das Dentin unter Wasserkühlung plan geschliffen (Siliziumcarbamid Schleifpapier P500, Hermes Schleifmittel GmbH; Schleifmaschine Roto-Pol34, Struers) und poliert.

Mithilfe einer speziell hergestellten Applikationshilfe wurde ein transparenter Acrylzylinder (Innendurchmesser: $3 \mathrm{~mm}$ ) senkrecht auf der plan geschliffenen Zahnoberfläche fixiert. Die Applikationshilfe besteht aus einer Metallbasis, auf der der Prüfkörper durch Markierungen zentral positioniert werden kann. Auf der Basis kann über drei Gewindeschrauben eine Metallplatte geführt werden, die eine Aussparung in der Mitte aufweist, in die der Acrylzylinder exakt hineinpasst. Durch Muttern kann die Metallplatte auf den Gewindeschrauben im gewünschten Abstand zu der Metallbasis gehalten werden und somit den Acrylzylinder mittig auf der Prüfkörperbasis fixieren (Abb. 4.1).

Der Acrylzylinder wurde bis zu einer maximalen Füllhöhe von $2 \mathrm{~mm}$ mit dem jeweiligen fließfähigen Komposit befüllt. Die Applikation der Komposite erfolgte strikt nach Herstellerangaben (Tabelle 4.1), wobei die selbstadhäsiven Komposite zunächst in einer initialen dünnen Schicht aktiv einmassiert wurden, bevor in einem zweiten Applikationsschritt die maximale Füllhöhe erreicht wurde. 


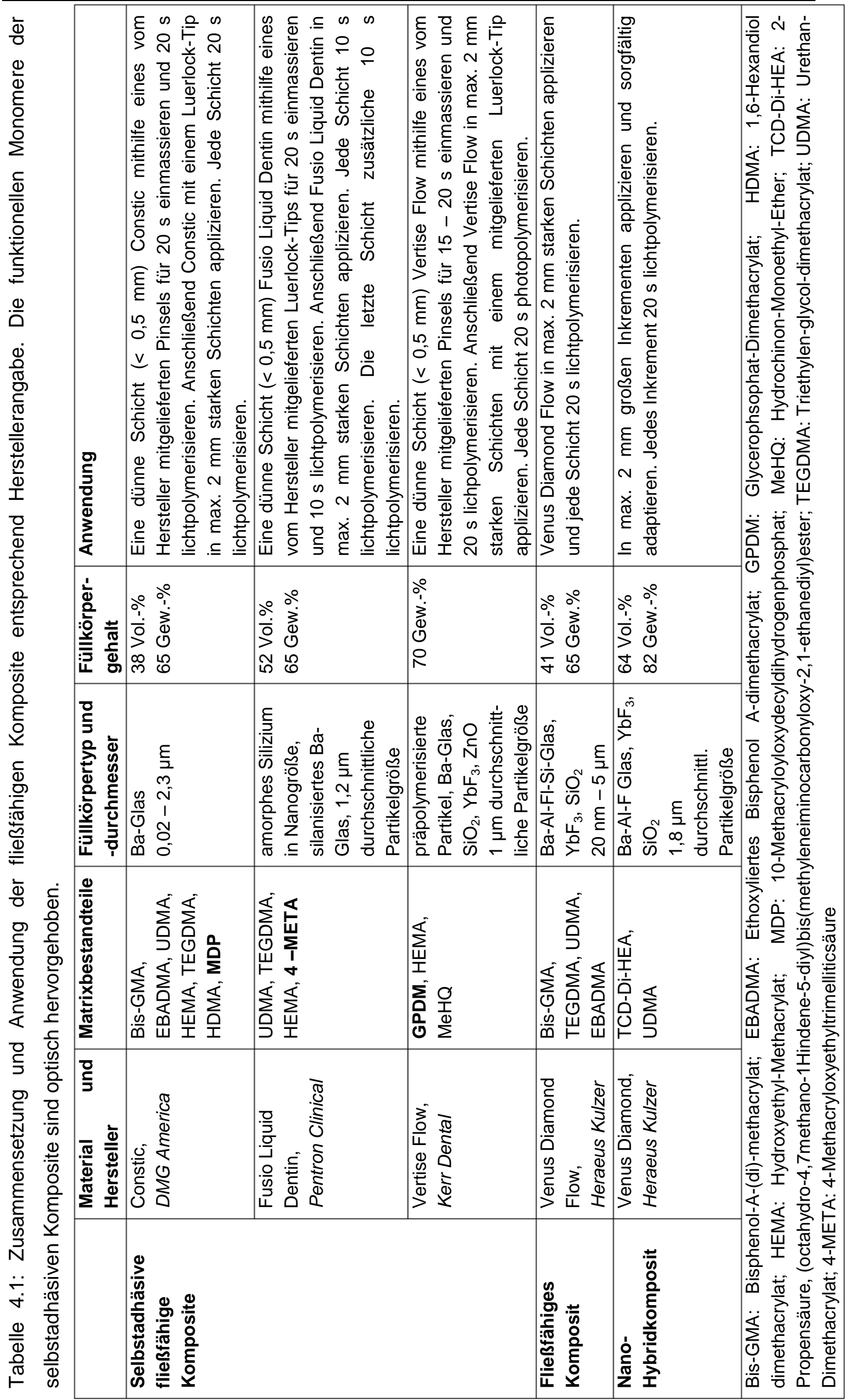


In der Kontrollgruppe wurde vor Applikation des Acrylzylinders ein Adhäsivsystem (Optibond FL, Kerr Dental) nach Herstellerangabe in Etch\&Rinse-Technik angewendet: Schmelzätzung $30 \mathrm{~s}$ und Dentinätzung $15 \mathrm{~s}$ mit $35 \%$ iger Phosphorsäure (Ultra-Etch, Ultradent Products Inc.); Primer $15 \mathrm{~s}$ einmassieren und $5 \mathrm{~s}$ mit einem Luftstrahl trocknen; Ädhäsiv $15 \mathrm{~s}$ einmassieren und $3 \mathrm{~s}$ mit einem Luftstrahl trocknen, $20 \mathrm{~s}$ lichtpolymerisieren (BA OPTIMA 10, B.A.International, Intensität $>800 \mathrm{~mW} / \mathrm{cm}^{2}$ ).

Nach Lichtpolymerisation (Polymerisationszeit nach Herstellerangabe, Tabelle 4.1) der fließfähigen Komposite wurden die Prüfkörper durch Lösen der Muttern vorsichtig aus der Applikationshilfe gelöst. Alle Zahnhartsubstanzprüfkörper wurden mittels Thermolastwechsel (5000 Zyklen, $5-55^{\circ} \mathrm{C}$ ) artifiziell gealtert (s.u.).

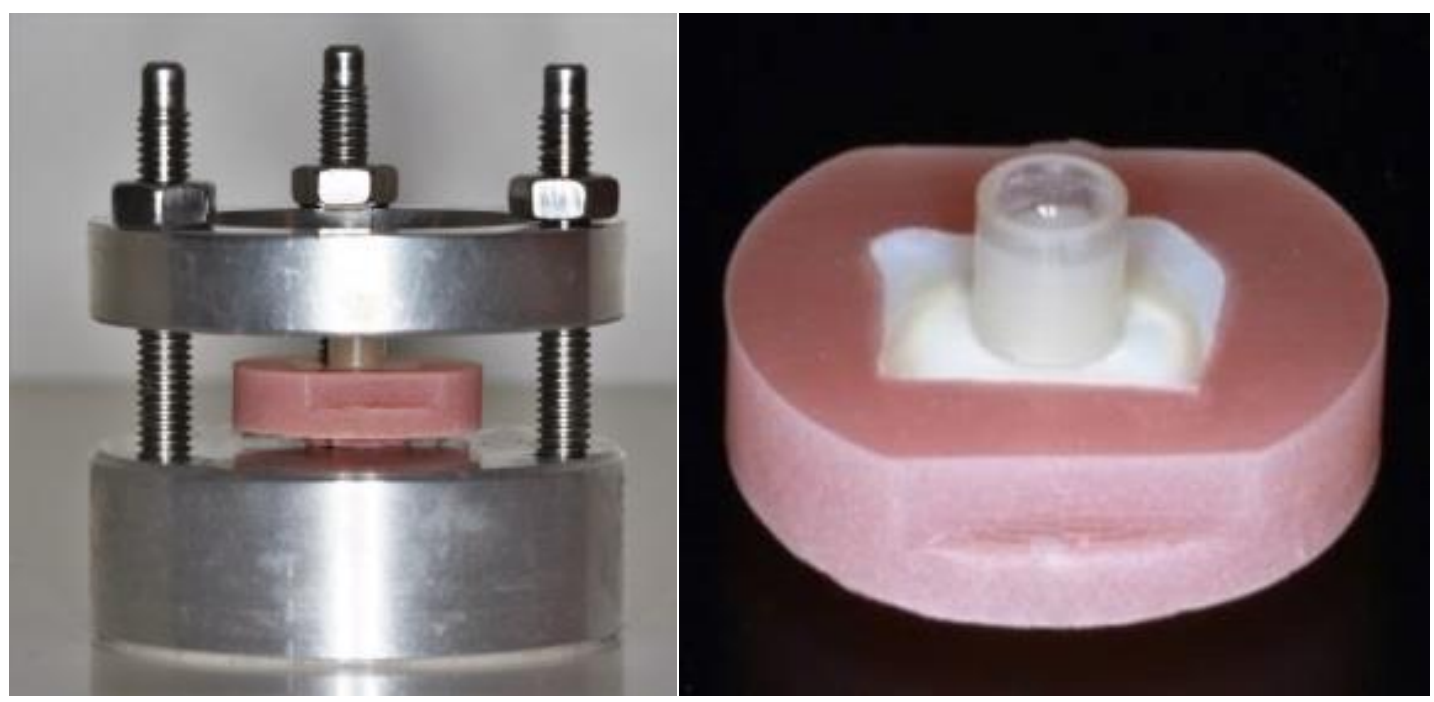

Abbildung 4.1: Applikationshilfe und Zahnhartsubstanzprüfkörper 


\subsection{Herstellung der Prüfkörper - Haftung auf Komposit}

Das Nano-Hybridkomposit (Venus Diamond A3, Heraeus Kulzer) wurde gemäß Herstellerangaben in Inkrementtechnik in den inneren Hohlraum $(6 \mathrm{~mm}$ Durchmesser) eines Metallringes appliziert. Jedes Inkrement wurde gut an den Metallring und ggf. an die anderen Inkremente adaptiert und nach Herstellerangabe $20 \mathrm{~s}$ lichtpolymerisiert. Die Prüfkörper wurden unter Wasserkühlung plan geschliffen und poliert (Siliziumcarbamid Schleifpapiere P800 - P1200 - P4000, Hermes Schleifmittel GmbH; Schleifmaschine Roto-Pol34, Struers). Falls die Kompositoberfläche nach dem Schleifvorgang makroskopisch sichtbare Unregelmäßigkeiten (z. B. Lufteinschlüsse) aufwies, wurde der Prüfkörper verworfen.

Anschließend erfolgte eine artifizielle Alterung im Thermolastwechselverfahren (5000 Zyklen, $5-55^{\circ} \mathrm{C}$ ).

Um verschiedene Möglichkeiten zur Konditionierung der Kompositoberflächen für eine Reparaturrestauration zu simulieren, erfolgte eine zufällige Aufteilung der Prüfkörper in vier Gruppen ( $n=128)$ :

1. Mechanisches Anrauen zur Simulation eines Diamantschleifers: Die Prüfkörper wurden unter Wasserkühlung angeraut (Siliziumcarbamid Schleifpapier P500), mit Wasser gereinigt und mithilfe eines Luftstrahls getrocknet.

2. Sandstrahlen mit $\mathrm{Al}_{2} \mathrm{O}_{3}$ : Die Prüfkörper wurden mithilfe eines Sandstrahlgerätes (CoJet ${ }^{\mathrm{TM}}$ Prep, $3 M$ Espe) im $45^{\circ}$ Winkel und $10 \mathrm{~mm}$ Abstand mit $\mathrm{Al}_{2} \mathrm{O}_{3}$-Sand (Airsonic Aluminium-Oxyd Pulver $50 \mu \mathrm{m}$, HAGER WERKEN, $15 \mathrm{~s}, 2,8$ bar) abgestrahlt. Die Sandreste wurden durch gründliches Abblasen mit einem Luftstrahl entfernt.

3. Silikatisierung/Silanisierung: Die Prüfkörper wurden mithilfe eines Sandstrahlgerätes (CoJet ${ }^{\mathrm{TM}}$ Prep, $3 \mathrm{M}$ Espe) im $45^{\circ}$ Winkel und $10 \mathrm{~mm}$ Abstand mit Silikat-beschichtetem $\mathrm{Al}_{2} \mathrm{O}_{3}$-Sand (CoJet ${ }^{\mathrm{TM}}$ Sand Blast Coating Agent $30 \mu \mathrm{m}, 3 \mathrm{M}$ Espe, $15 \mathrm{~s}, 2,8$ bar) abgestrahlt. Die Sandreste wurden durch gründliches Abblasen mit einem Luftstrahl entfernt. Im Anschluss wurde eine Silan-Lösung (Espe ${ }^{\mathrm{TM}}$ Sil, 3M Deutschland) appliziert und die Überschüsse mit Luft verblasen.

4. Keine Oberflächenbearbeitung: Nach Trocknung mithilfe eines Luftstrahls wurden die Prüfkörper dieser Gruppe keiner weiteren Oberflächenbearbeitung unterzogen. 
Anschließend wurde entweder das konventionelle fließähige Komposit Venus Diamond Flow oder eines der fließfähigen selbstadhäsiven Komposite aufgetragen. Zuvor wurde entweder ein Adhäsivsystem (Optibond FL, Kerr Dental, Anwendung gemäß Herstellerangaben s.o.) appliziert oder auf eine weitere Konditionierung mittels eines Adhäsivsystems verzichtet.

Die fließfähigen Komposite wurden nach Herstellerangaben in einer Füllhöhe von max. $2 \mathrm{~mm}$ in den Acrylzylinder (Abb. 4.2) appliziert und lichtpolymerisiert. In jeder Untergruppe wurden $n=16$ Prüfkörper verwendet. Alle Prüfkörper wurden nun erneut einem Thermolastwechsel (5000 Zyklen, $\left.5-55^{\circ} \mathrm{C}\right)$ unterzogen.

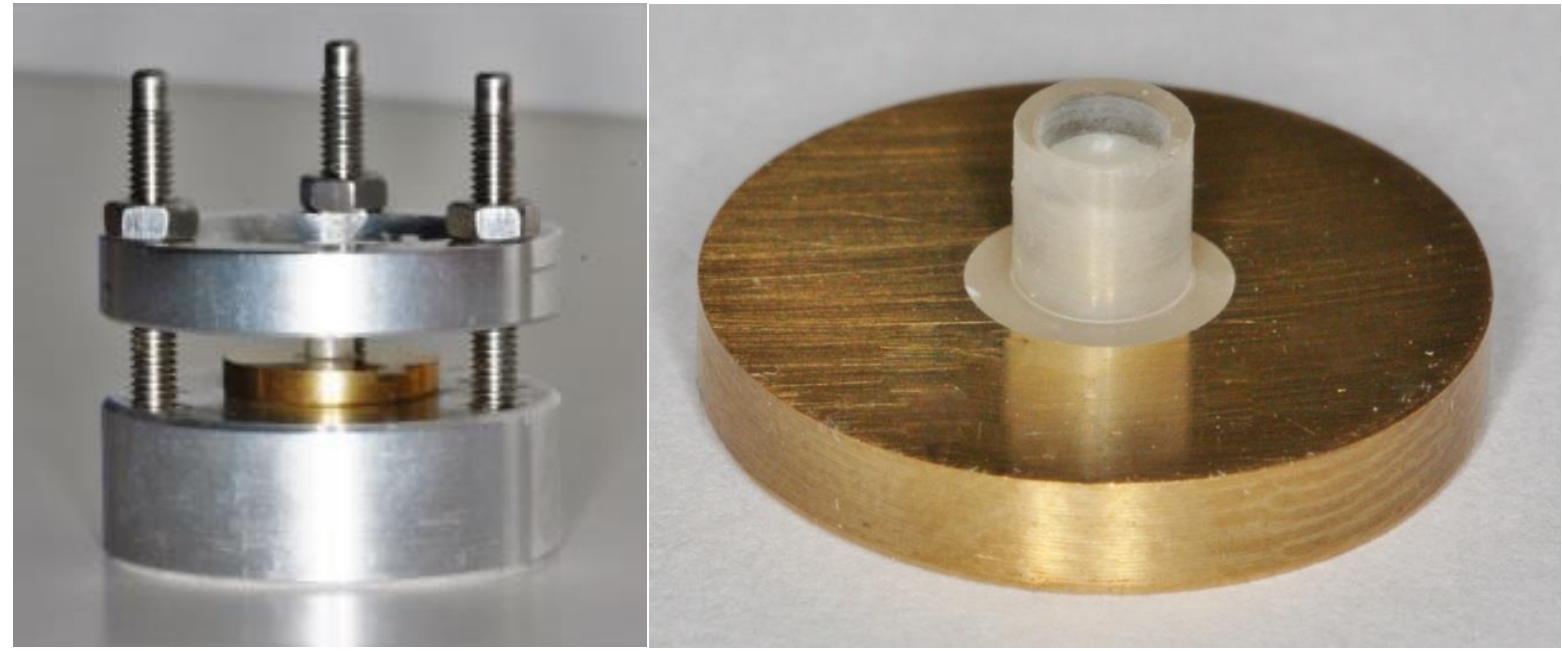

Abbildung 4.2: Applikationshilfe und Nano-Hybridkompositprüfkörper 


\subsection{Thermisches Lastwechselverfahren}

Der verwendete thermische Lastwechsler (WILLYTEC THERMOCYCLER V 2.9, Gebrüder Haake $\mathrm{GmbH}$ ) besteht aus einem Schwenkarm zwischen zwei Tauchwannen (Haake W15, Gebrüder Haake GmbH) (Abb. 4.3). Beide Tauchwannen sind mit einem programmierbaren Thermostat ausgestattet (Haake DC1, Gebrüder Haake $\mathrm{GmbH}$ ), zudem ist eine Wanne mit einem Durchflusskühler (Haake EK 20, Gebrüder Haake $\mathrm{GmbH}$ ) verbunden. Für den Versuch wurde in der einen Wanne eine Wassertemperatur von $55{ }^{\circ} \mathrm{C}$, in der anderen eine Wassertemperatur von 5 ${ }^{\circ} \mathrm{C}$ gehalten. Die Prüfkörper wurden in einer Haltevorrichtung in einem Sieb am Schwenkarm befestigt und wurden so positioniert, dass sie nicht in gegenseitigem Kontakt standen und für ausreichend Durchfluss des Wassers der Tauchwannen gesorgt war. Zum Durchlaufen eines Zyklus tauchte der Schwenkarm die Prüfkörper zunächst $20 \mathrm{~s}$ in das $5^{\circ} \mathrm{C}$ kalte Wasser, es folgten $5 \mathrm{~s}$ Abtropfzeit. Anschließend wurden die Prüfkörper $20 \mathrm{~s}$ in das $55{ }^{\circ} \mathrm{C}$ warme Wasser getaucht, es folgten weitere $5 \mathrm{~s}$ Abtropfzeit. Ein Zyklus umfasste eine Zeitspanne von $61 \mathrm{~s}$, für die Gesamtzyklenzahl von 5000 wurden die Prüfkörper folglich 3,5 Tage dem Thermocycling ausgesetzt.

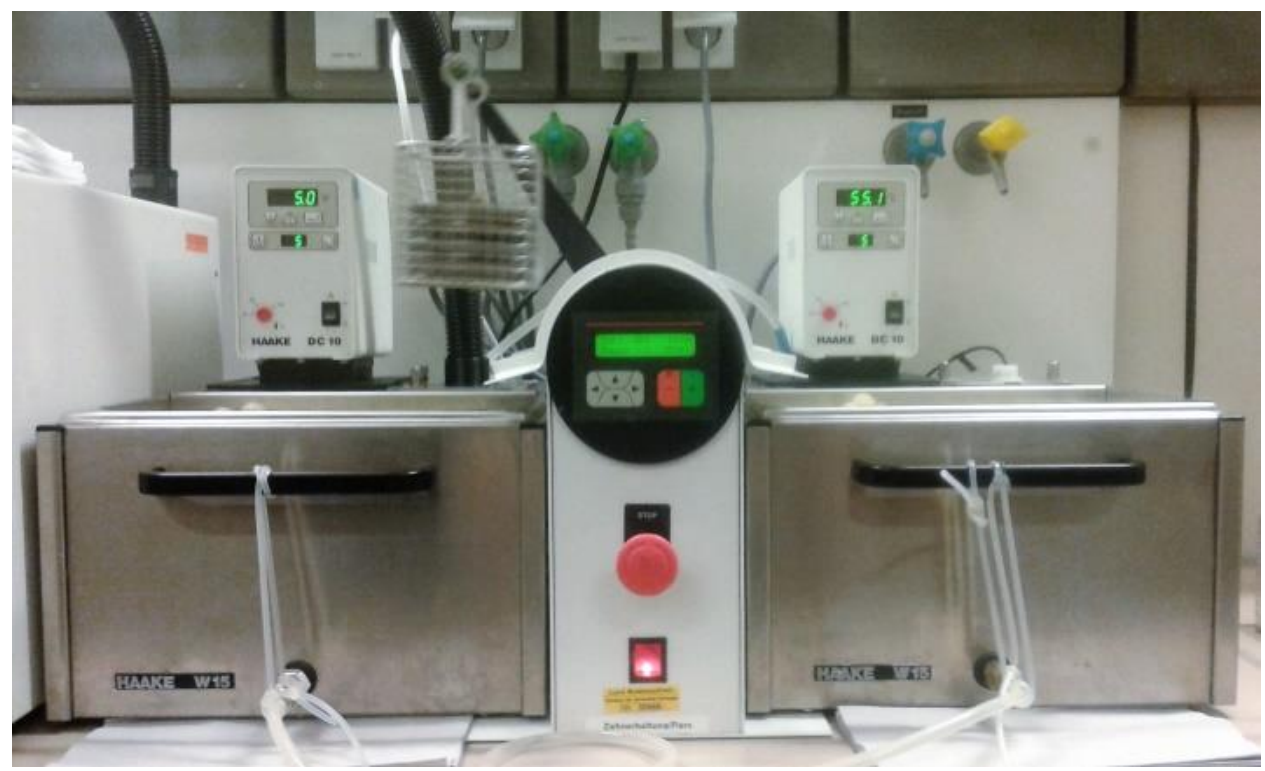

Abbildung 4.3: Thermischer Lastwechsler: In der linken Tauchwanne herrscht eine Temperatur von $5{ }^{\circ} \mathrm{C}$, in der rechten Tauchwanne eine Temperatur von $55^{\circ} \mathrm{C}$. Am Schwenkarm ist eine Halterung mit einem Sieb befestigt, in dem sich die Prüfkörper befinden und abwechselnd in die Bäder getaucht werden. 


\subsection{Versuchsdurchführung}

\subsubsection{Scherfestigkeitsanalyse}

Die Scherfestigkeitsanalyse wurde mit einer universellen Testmaschine (Materialprüfmaschine 1446, Zwick $\mathrm{GmbH}$ \& Co. KG) durchgeführt. Vor Beginn der Messungen wurde die Maschine von der Firma Zwick kalibriert.

Alle Prüfkörper wurden in einen speziell angefertigten Halter gespannt (Abb. 4.4) und dabei so fixiert, dass der Scherstempel der universellen Testmaschine genau senkrecht möglichst nah am Übergang zwischen Zylinder und Prüfkörperbasis ansetzen konnte. Der Scherstempel wurde mit gleichmäßiger Geschwindigkeit (1 $\mathrm{mm} / \mathrm{min}$ ) appliziert. Dabei wurde die Kraft so lange erhöht, bis es zu einer Abscherung des Zylinders von der Prüfkörperbasis kam. Die Kraft zum Zeitpunkt der Abscherung wurde registriert (Software: testXpert V12.1, Zwick GmbH \& Co. $K G)$.

Einige der Komposit-befüllten Zylinder lösten sich schon während des Thermolastwechsels von den Prüfkörperbasen, in diesem Fall wurde die Scherfestigkeit als $0 \mathrm{MPa}$ definiert.

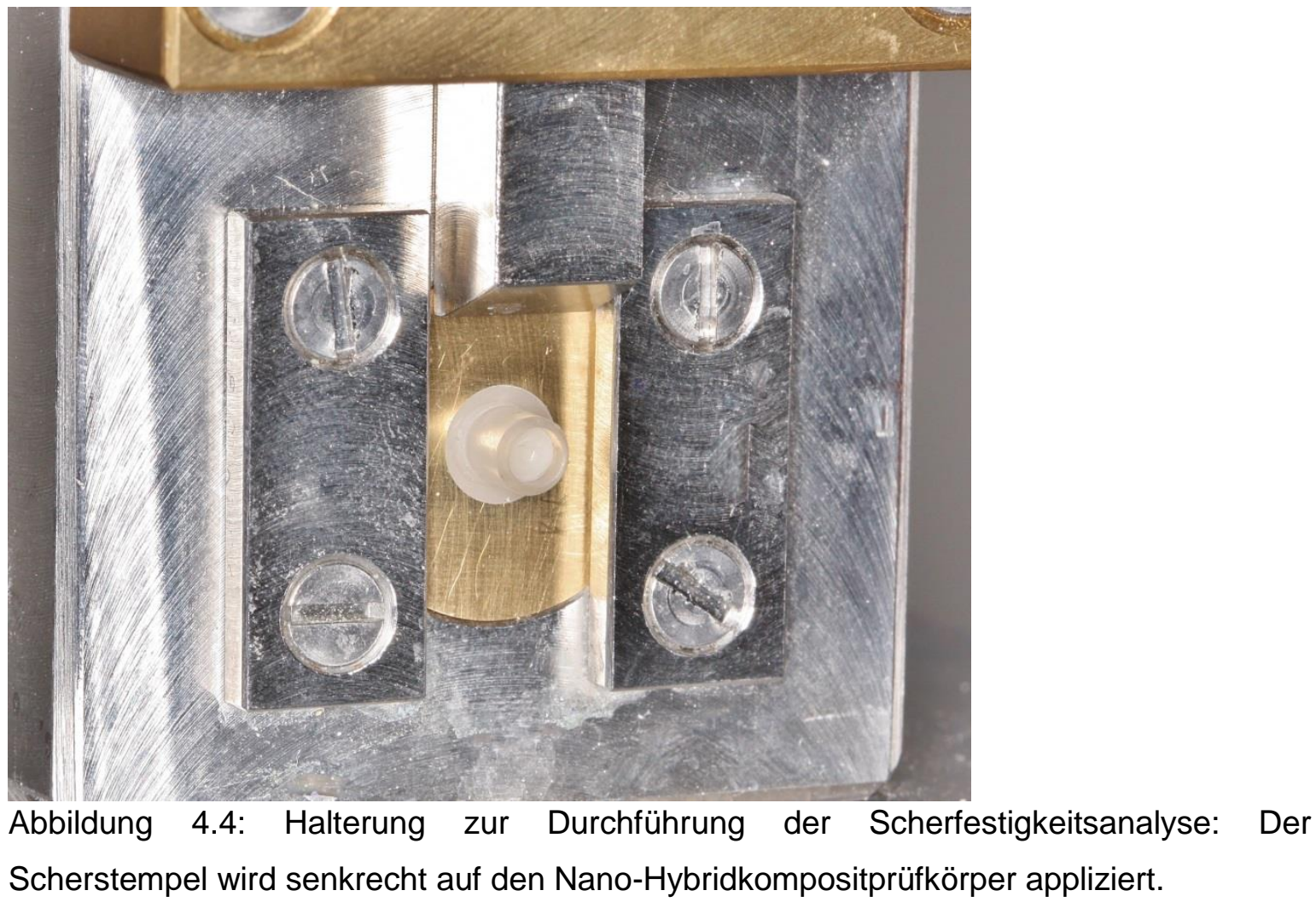




\subsubsection{Bruchstellenanalyse}

Im Anschluss wurden alle Bruchstellen mikroskopisch (Stemi SV 11, Zeiss Deutschland) bei 1,6facher Vergrößerung analysiert. Dabei wurde zwischen drei verschiedenen Bruchtypen unterschieden (Abb. 4.5):

Kohäsiver Bruchtyp: Bruch ausschließlich innerhalb der Prüfkörperbasis (Schmelz, Dentin oder Nano-Hybridkomposit) oder innerhalb des fließfähigen Komposits.

Adhäsiver Bruchtyp: Bruchstelle zwischen fließfähigem Komposit und Prüfkörperbasis.

Gemischter Bruchtyp: enthält sowohl Anteile eines kohäsiven als auch eines adhäsiven Bruchtyps.

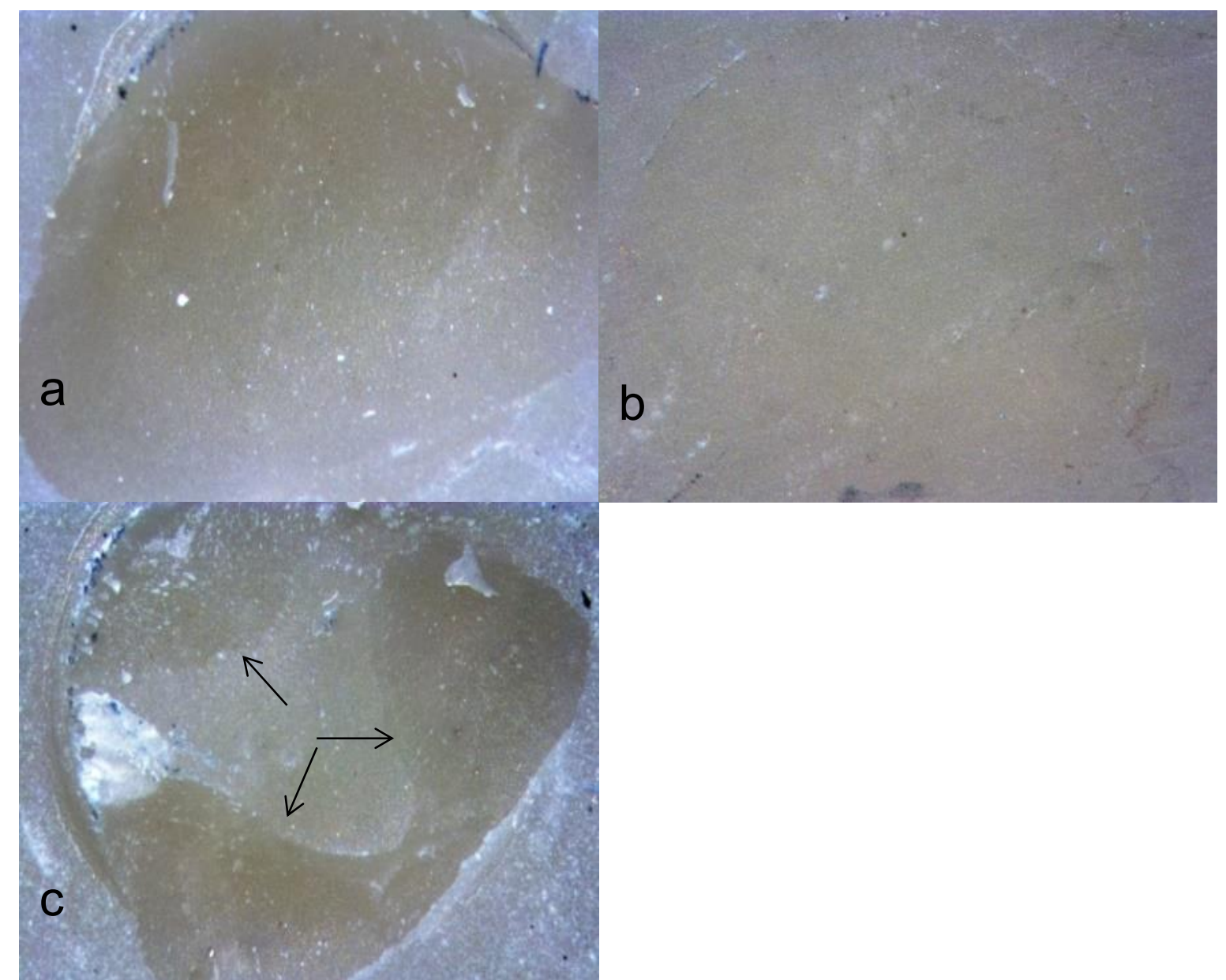

Abbildung 4.5: Bruchstellenanalyse: (a) kohäsiver Bruchtyp: Bruch ausschließlich im Nano-Hybridkomposit, (b) adhäsiver Bruchtyp: Bruch zwischen appliziertem fließfähigem Komposit und Nano-Hybridkomposit, (c) gemischter Bruchtyp (Die Pfeile markieren die Grenzfläche zwischen adhäsivem und kohäsivem Bruchmuster.) 


\subsection{Statistische Auswertung}

Zunächst wurden die ermittelten Scherfestigkeiten mittels Quantil-Quantil-Plots auf Normalverteilung getestet. Da $80 \%$ der Subgruppen normalverteilt waren, wurde für die weitere statistische Analyse eine Normalverteilung der Daten angenommen.

Die statistische Auswertung der Scherfestigkeiten erfolgte mittels 3-faktoriellen und einfaktoriellen ANOVAs und Bonferroni-post-hoc-Tests $(p<0,05)$. Zusätzlich wurden die Weibull-Parameter (Weibull-Modul $m$ und charakteristische Festigkeit ơ) mithilfe der Maximum-Likelihood-Schätzung bei einem 95\% Konfidenzintervall berechnet.

Unterschiede der verschiedenen Untergruppen hinsichtlich ihrer Bruchtypenverteilung wurden mittels Chi'-Test ausgewertet $(p<0,05)$.

Alle statistischen Auswertungen wurden mit der Software Statistica (Version 12, StatSoft) durchgeführt. 


\section{Ergebnisse}

\subsection{Scherfestigkeitsanalyse Zahnhartsubstanz}

Die Scherfestigkeiten der selbstadhäsiven Komposite auf Zahnhartsubstanz waren sehr niedrig (Schmelz: < $5 \mathrm{MPa}$; Dentin: < $3 \mathrm{MPa}$ ) und signifikant geringer als die von Venus Diamond Flow (Schmelz: 13,0 \pm 5,1 MPa, Dentin: 11,2 \pm 6,3 MPa), wobei zwischen Schmelz und Dentin keine signifikanten Unterschiede feststellbar waren (Tabelle 5.1).

Tabelle 5.1: Haftfestigkeiten (MPa, Mittelwert \pm Standardabweichung) der fließfähigen Komposite auf Schmelz und Dentin

\begin{tabular}{|c|c|c|}
\hline & Schmelz & Dentin \\
\hline Constic & $4,4 \pm 4,7^{\mathrm{a}}$ & $0,4 \pm 0,9^{\mathrm{a}}$ \\
\hline Fusio Liquid Dentin & $3,1 \pm 4,8^{\mathrm{a}}$ & $2,7 \pm 3,1^{\mathrm{a}}$ \\
\hline Vertise Flow & $1,9 \pm 2,5^{\mathrm{a}}$ & $0,9 \pm 1,4^{\mathrm{a}}$ \\
\hline Venus Diamond Flow (Kontrolle) & $13,0 \pm 5,1^{\mathrm{b}}$ & $11,2 \pm 6,3^{\mathrm{b}}$ \\
\hline
\end{tabular}

Signifikante Unterschiede zwischen den verschiedenen fließfähigen Kompositen innerhalb einer Zahnhartsubstanz sind mit unterschiedlichen Kleinbuchstaben markiert.

Hinsichtlich ihrer Weibull-Module wiesen die selbstadhäsiven Komposite auf Schmelz und Dentin kaum Unterschiede auf (Tabelle 5.2). Ihre charakteristische Festigkeit erreichte Werte von 1,4 bis 3,9 MPa auf Schmelz und von 0,3 bis 2,4 $\mathrm{MPa}$ auf Dentin. Die Kontrollgruppe erreichte sowohl für den Weibull-Modul (Schmelz: 2,8; Dentin: 1,9) als auch für die charakteristische Festigkeit (Schmelz: 14,6 MPa; Dentin 12,6 MPa) höhere Werte als die selbstadhäsiven Komposite (Tabelle 5.2). 
Tabelle 5.2: Weibull-Parameter (95\% Konfidenzintervalle) der fließfähigen Komposite auf Schmelz und Dentin

\begin{tabular}{|c|c|c|c|c|}
\hline \multirow{2}{*}{} & \multicolumn{2}{|c|}{ Weibull-Modul m (95\% KI) } & $\begin{array}{r}\text { Charakteristische Festigkeit o'0 } \\
(95 \% \mathrm{KI})\end{array}$ \\
\cline { 2 - 5 } & Schmelz & Dentin & Schmelz & Dentin \\
\hline Constic & $0,8(0,5 ; 1,2)$ & $0,7(0,5 ; 1,0)$ & $3,9(2,0 ; 7,5)$ & $0,3(0,2 ; 0,7)$ \\
\hline $\begin{array}{c}\text { Fusio Liquid } \\
\text { Dentin }\end{array}$ & $0,7(0,5 ; 1,0)$ & $0,8(0,5 ; 1,2)$ & $2,5(1,2 ; 5,1)$ & $2,4(1,3 ; 4,6)$ \\
\hline $\begin{array}{c}\text { Vertise Flow } \\
\text { Venus Diamond } \\
\text { Flow (Kontrolle) }\end{array}$ & $0,7(0,5 ; 1,0)$ & $0,7(0,5 ; 1,0)$ & $1,4(0,7 ; 3,1)$ & $0,7(0,3 ; 1,5)$ \\
\hline
\end{tabular}

\subsection{Scherfestigkeitsanalyse Komposit}

Die Haftung der Materialien auf dem Nano-Hybridkomposit Venus Diamond war signifikant abhängig von der Art der Vorbehandlung. Die signifikant höchsten Reparaturhaftwerte wurden nach Silikatisierung und Silanisierung gemessen, gefolgt von der Verwendung von Aluminiumoxid-Sand sowie mechanischem Anrauen. In allen Gruppen waren die Haftwerte ohne vorhergehende Vorbehandlung am niedrigsten.

Die Applikation des Adhäsivsystems verbesserte die Scherfestigkeit des konventionellen, aber nicht der selbstadhäsiven Komposite signifikant. Insgesamt war die Haftung von Venus Diamond Flow auf dem Nano-Hybridkomposit signifikant besser als die der selbstadhäsiven Komposite. Fusio Liquid Dentin erreichte jedoch in einigen Untergruppen vergleichbar gute Werte wie die Kontrollgruppe Venus Diamond Flow in Kombination mit einem Adhäsivsystem (Tabelle 5.3).

Die Reparaturhaftwerte der selbstadhäsiven Komposite auf Nano-Hybridkomposit waren besser als die Haftwerte auf Zahnhartsubstanz.

Die Weibull-Module der Nano-Hybridkompositprüfkörper variierten zwischen 1,3 und 5,9 für die selbstadhäsiven Komposite und erreichten Werte bis zu 9,7 in der Kontrollgruppe (Tabelle 5.4). 
Die charakteristische Festigkeit aller fließfähigen Komposite wurde durch die mechanische Bearbeitung der Reparaturoberflächen im Vergleich zu unbearbeiteten Oberflächen verbessert. Dabei erreichten die selbstadhäsiven Komposite charakteristische Festigkeiten von 6,3 bis 24,3 MPa. In der Kontrollgruppe wurden Werte bis zu 25,7 MPa erzielt. 


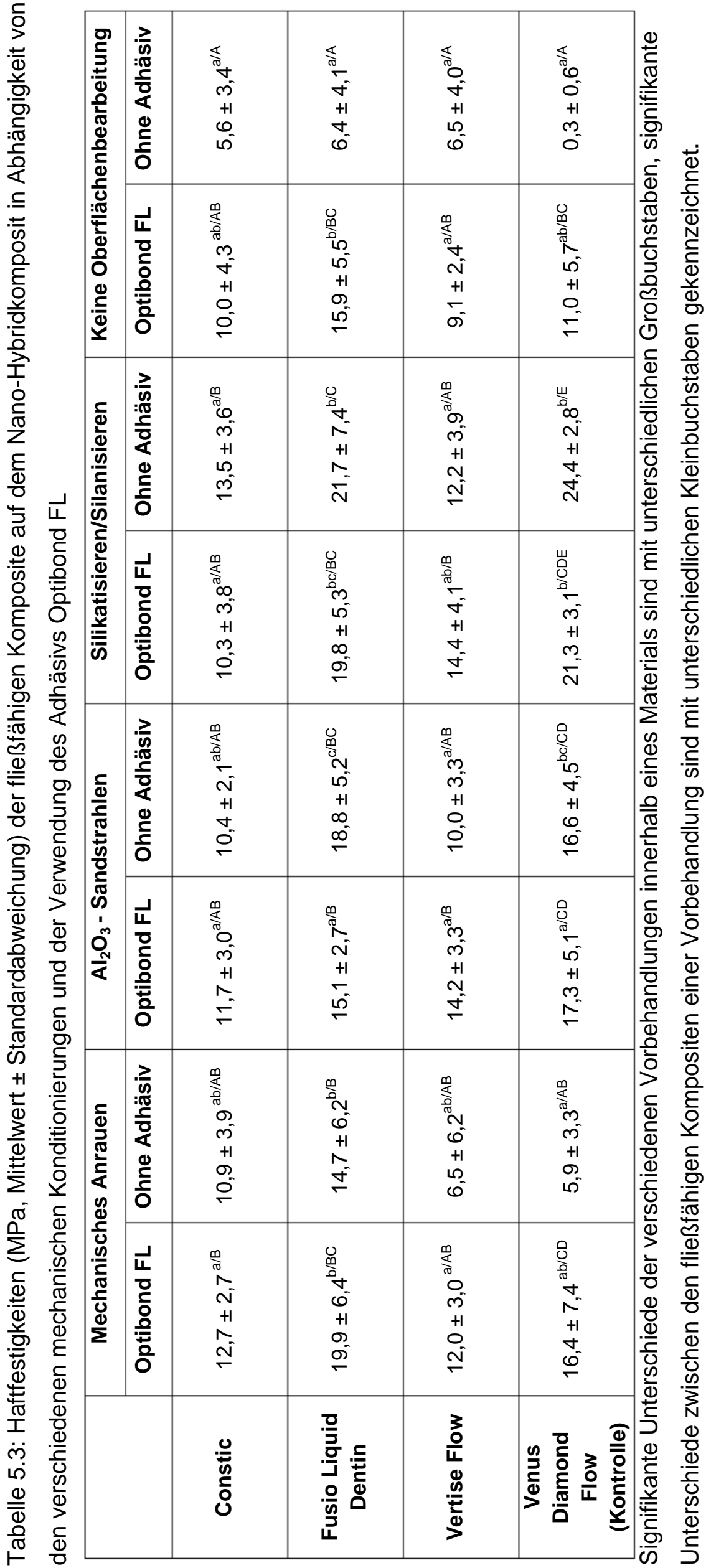




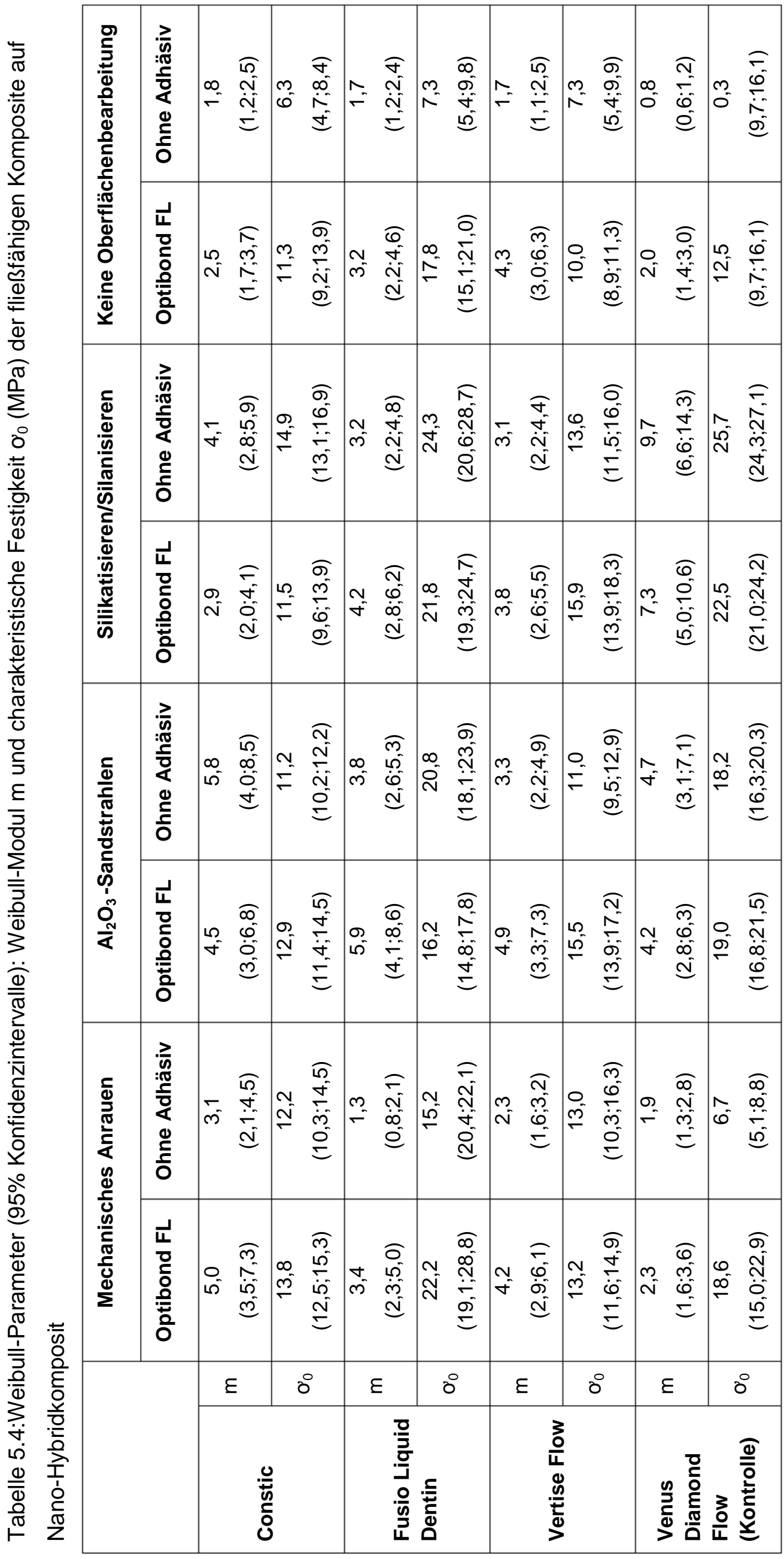




\subsection{Bruchstellenanalyse Zahnhartsubstanz}

Die Verteilung der Bruchtypen unterschied sich sowohl auf den Zahnhartsubstanzen als auch auf dem Nano-Hybridkomposit in allen Gruppen signifikant. Auf Schmelz und Dentin konnten kohäsive Bruchtypen nur in der Kontrollgruppe beobachtet werden, wobei auf Schmelz eine größere Anzahl an kohäsiven Brüchen und eine geringere Anzahl an adhäsiven Bruchmustern erzielt wurde als auf Dentin. Hinsichtlich der selbstadhäsiven fließfähigen Komposite konnten sowohl auf Schmelz als auch auf Dentin lediglich adhäsive und gemischte Versagensmuster festgestellt werden (Tabelle 5.5).

Tabelle 5.5: Versagensmuster (\%) der fließähigen Komposite auf Zahnhartsubstanz

\begin{tabular}{|c|c|c|c|c|c|c|}
\hline & \multicolumn{3}{|c|}{ Schmelz } & \multicolumn{3}{c|}{ Dentin } \\
\cline { 2 - 7 } & \multicolumn{3}{|c|}{ Bruchmodus } & \multicolumn{3}{c|}{ Bruchmodus } \\
\cline { 2 - 7 } & Kohäsiv & Adhäsiv & Gemischt & Kohäsiv & Adhäsiv & Gemischt \\
\hline Constic & 0 & 81 & 19 & 0 & 81 & 19 \\
\hline Fusio Liquid Dentin & 0 & 38 & 62 & 0 & 100 & 0 \\
\hline Vertise Flow & 0 & 69 & 31 & 0 & 69 & 31 \\
\hline $\begin{array}{c}\text { Venus Diamond Flow } \\
\text { (Kontrolle) }\end{array}$ & 31 & 6 & 63 & 6 & 19 & 75 \\
\hline
\end{tabular}

\subsection{Bruchstellenanalyse Komposit}

Auch bei der simulierten Reparatur des Nano-Hybridkomposits trat die Mehrzahl der kohäsiven Brüche in der Kontrollgruppe auf. Hier konnten jedoch auch bei den selbstadhäsiven Kompositen in einigen Untergruppen kohäsive Bruchtypen beobachtet werden. Die Durchführung einer mechanischen Oberflächenkonditionierung sowie die Applikation des Adhäsivsystems verringerte das Auftreten von adhäsiven Bruchmustern für alle fließfähigen Komposite (Tabelle $5.6)$. 
Tabelle 5.6: Versagensmuster (\%) der fließfähigen Komposite auf Nano-Hybridkomposit in Abhängigkeit von der Oberflächenkonditionierung und der Verwendung des Adhäsivsystems

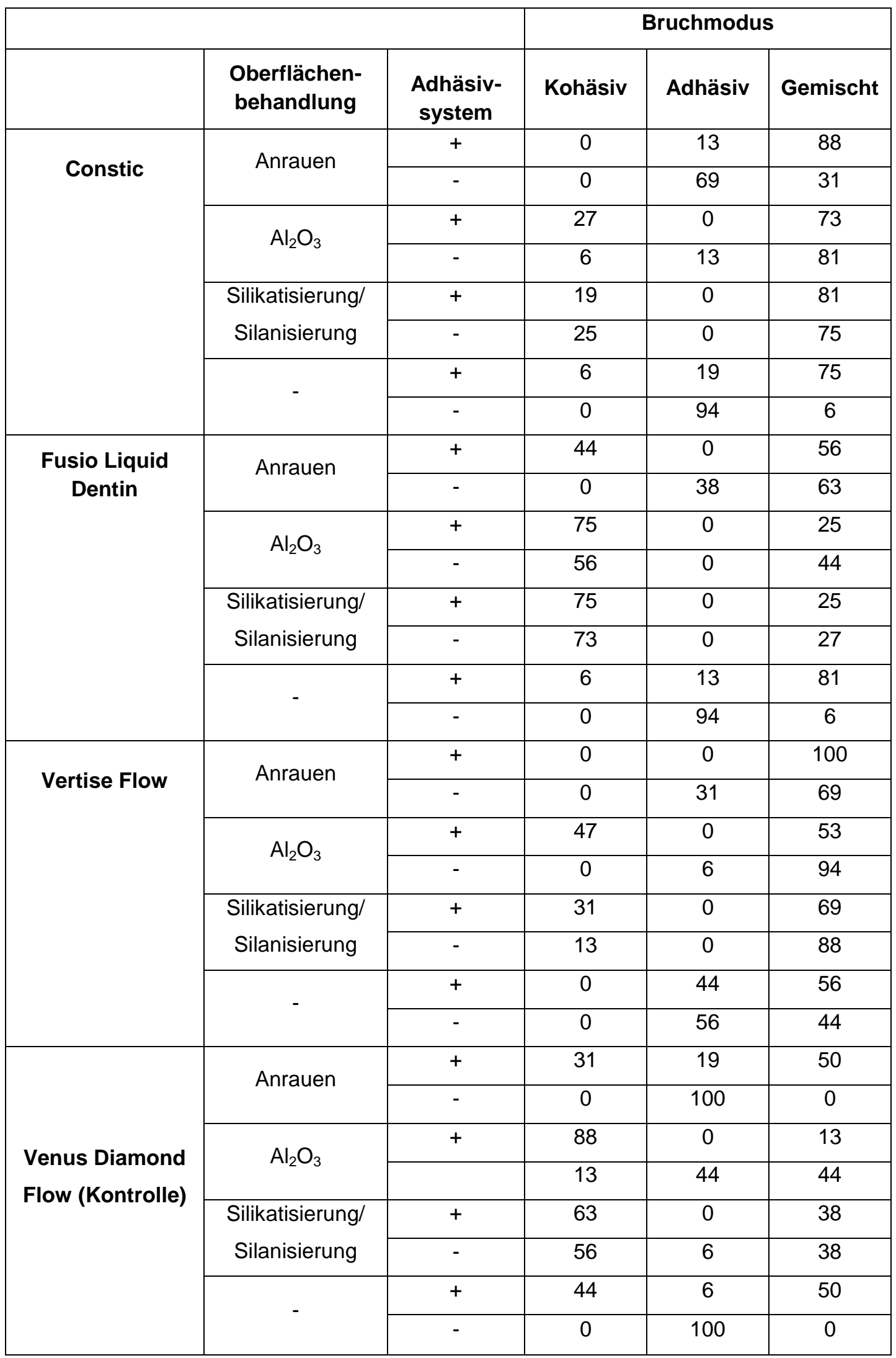




\section{Diskussion}

\subsection{Material und Methode}

Bei der vorliegenden Studie handelt es sich um eine In-vitro-Studie, ihre Ergebnisse basieren also rein auf Simulationsprozessen im Labor und sind somit nicht ohne Einschränkungen auf klinische Situationen übertragbar. In-vitro-Studien lassen sich jedoch im Gegensatz zu In-vivo-Untersuchungen leichter standardisieren und sind meist ethisch unbedenklicher. Für dentale Materialien lassen Laboruntersuchungen eine grundsätzliche Beurteilung der klinischen Anwendbarkeit zu (Peumans et al. 2005; Frankenberger et al. 2007; Hebling et al. 2007), bevor aufwendigere In-vivo-Studien durchgeführt werden. Die vorliegende Arbeit basiert auf dem Konzept, dass Komposite und Adhäsivsysteme, die in vitro hohe Haftwerte erreichen können, auch in vivo eine längere Überlebensdauer aufweisen, als wenn schon unter Laborbedingungen schlechte Materialeigenschaften ermittelt wurden (Van Meerbeek et al. 2003).

\subsubsection{Materialauswahl}

Die Analyse der Haftwerte auf Zahnhartsubstanz wurde an extrahierten kariesund füllungsfreien humanen permanenten Zähnen durchgeführt. Potenzielle zahnund patientenbezogene Unterschiede bezüglich des Alters, der Zusammensetzung und Qualität der Zahnhartsubstanz wurden durch eine ausreichend große Anzahl an Prüfkörpern angeglichen. Da restaurationsbedürttige Defekte in der Regel Schmelz- und Dentinoberflächen einschließen, wurden beide Substrate untersucht. Zur Simulation einer reparaturbedürftigen Restauration wurden die Prüfkörperbasen im zweiten Versuch aus dem Nano-Hybridkomposit Venus Diamond hergestellt, da es sich dabei um ein häufig für die Anfertigung direkter Restaurationen verwendetes konventionelles Komposit handelt.

Zum Zeitpunkt der Versuchsdurchführung wurden alle auf dem Markt erhältlichen und für die Anfertigung direkter Restaurationen geeigneten selbstadhäsiven Komposite untersucht. Als Kontrollgruppe diente Venus Diamond Flow, ein in der klinischen Anwendung seit langem etabliertes konventionelles fließfähiges Komposit, welches mit dem Etch\&Rinse-Adhäsivsystem Optibond FL verwendet wurde, das als Goldstandard in der Adhäsivtechnik gilt (Van Meerbeek et al. 2003). 


\subsubsection{Simulation der Kompositreparatur}

Es gibt verschiedene Möglichkeiten, Kompositrestaurationen für eine Reparatur vorzubereiten. Ein optimales Reparaturprotokoll liegt jedoch nicht vor (Imbery et al. 2014). Daher wurden in der vorliegenden Studie die gängigsten Reparaturprotokolle (Anrauen der Reparaturoberfläche mittels Diamantschleifer; Silikatisierung und Silanisierung der Reparaturoberfläche; Abstrahlen der Reparaturoberfläche mit $\mathrm{Al}_{2} \mathrm{O}_{3}$-Sand) angewendet.

Für die Simulation des Anrauens mit einem rotierenden Diamantschleifer wurde Siliziumkarbidschleifpapier mit einer Korngröße von 30,2 \pm 1,5 $\mu \mathrm{m}$ unter Wasserkühlung verwendet. Durch einen konstanten Anpressdruck und eine einheitliche Bearbeitungszeit konnte so für alle Prüfkörper eine annähernd identisch angeraute Oberfläche erzielt werden.

Die Anwendung des Sandstrahlers mit $\mathrm{Al}_{2} \mathrm{O}_{3}-$ Sand und die Silikatisierung erfolgten laut Herstellerangaben und nach Empfehlungen vorhergehender Studien (Wiegand et al. 2012; Özcan et al. 2013) standardisiert bei einem Abstand von 10 $\mathrm{mm}$, einem Druck von 2,8 bar und einem Winkel von $45^{\circ}$. Der veränderte Anstellwinkel von $45^{\circ}$ (der Hersteller empfiehlt $90^{\circ}$ ) führte in einer Studie zu verbesserten Haftwerten, sowohl bei der Silikatisierung als auch bei der Anwendung des $\mathrm{Al}_{2} \mathrm{O}_{3}$-Sandstrahlers (Özcan et al. 2013).

Im Anschluss an die Silikatisierung wurde gemäß Herstellerangaben eine SilanLösung aufgetragen. Diese führt zum einen zu einer verbesserten Benetzbarkeit, zum anderen beeinflusst sie die chemische Bindung an anorganische Füllerpartikel und die Kompositmatrix (Rodrigues Jr. et al. 2009; Maneenut et al. 2011).

Niedrigvisköse Adhäsivsysteme können die Mikrounebenheiten der Reparaturoberfläche sehr gut benetzen. Ihre Anwendung resultierte in verbesserten Reparaturhaftwerten für konventionelle Komposite (Imbery et al. 2014). Um zu ermitteln, ob selbstadhäsive Komposite ebenfalls diese Fähigkeit aufweisen, wurden in der vorliegenden Studie die Reparaturhaftwerte sowohl nach vorheriger Konditionierung mittels Adhäsivsystem als auch ohne eine zusätzliche Konditionierung getestet. 


\subsubsection{Thermisches Lastwechselverfahren}

In der Mundhöhle eingesetzte Materialien müssen verschiedensten Belastungen widerstehen, um eine Langzeitstabilität zu erreichen. So kommt es neben der mechanischen Belastung beim Kauen auch durch chemische und thermische Belastungen während der Nahrungsaufnahme zu Alterungs- und Degradationsprozessen, die bei Kompositfüllungen zu Undichtigkeiten und Randspaltbildungen mit Entstehung von Sekundärkaries und Randverfärbungen führen können (Amaral et al. 2007). Diese Prozesse sind für eine komplette Umsetzung in vitro zu komplex, es wurden jedoch vereinfachte Verfahren entwickelt, mit denen Alterungsprozesse simuliert werden können. Eine weit verbreitete Methode stellt dabei die artifizielle Alterung im Thermolastwechselverfahren dar (Brendeke und Özcan 2007; Wiegand et al. 2012). Der Thermolastwechsel führt zu sich wiederholenden Kontraktionen und Expansionen an der Grenzfläche zwischen Prüfkörperbasis und appliziertem Restaurationsmaterial (Amaral et al. 2007). In verschiedenen Studien wurde nach Durchführung eines Thermolastwechselverfahrens eine Verschlechterung der Haftwerte von Kompositen festgestellt (Rinastiti et al. 2011; Goracci et al. 2013; Brueckner et al. 2017). Daher wurden auch in der vorliegenden Studie die Scherfestigkeiten der Komposite erst nach artifizieller Alterung im Thermolastwechselverfahren gemessen. Dafür wurde die nach ISO TS 11405 (International Organization for Standardization 2003) empfohlene Durchführung des Thermolastwechselverfahrens von 500 Zyklen abgewandelt, da diese für zu gering gehalten wird um einen realistischen Alterungsprozess zu simulieren (Brueckner et al. 2017). Die in dieser Studie angewendeten 5000 Zyklen simulieren zwar noch keine klinische Langzeitanwendung (10.000 Zyklen werden als äquivalent zu einem Jahr klinischer Anwendung angesehen (Gale und Darvell 1999)), eignen sich aber zur Unterscheidung, ob Materialien den Verhältnissen in der Mundhöhle widerstehen können oder dazu nicht in der Lage sind.

Restaurationen befinden sich meist schon eine längere Zeit in situ, bevor eine Reparatur nötig wird. Daher wurden die Nano-Hybridkompositprüfkörper vor Durchführung der Reparatursimulation schon ein erstes Mal artifiziell gealtert.

Weitere In-vitro-Modelle für die Simulation von Alterungs- und Degradationsprozessen in der Mundhöhle stellen die pH-Wechselbelastung, die mechanische Belastung sowie die Lagerung in Wasser oder Nahrungsmittel- 
simulierenden Lösungen dar.

Die am häufigsten angewendete Simulationsmethode stellt hierbei die Lagerung in Wasser dar (Hashimoto et al. 2003; Carrilho et al. 2004). Durch Hydrolyse kommt es dabei zu morphologischen Änderungen der Kollagenstrukturen im Dentin sowie der makroskopischen und mikroskopischen Kompositstrukturen, was zu einer Verminderung der Haftwerte führen kann (Hashimoto et al. 2003). Eine alleinige Lagerung in Wasser als Alterungssimulation lässt jedoch die Komplexität der in der Mundhöhle ablaufenden Degradationsprozesse, wie z. B. thermische und physikalische Faktoren außer Acht (Amaral et al. 2007).

Eine weitere Methode der artifiziellen Alterung stellt die Simulation der in der Mundhöhle ablaufenden mechanischen Belastung dar. Hier wird eine Verschlechterung der Haftwerte durch Ermüdung der Materialien angestrebt. Verschiedene Autoren kamen bei dieser Alterungsmethode jedoch zu stark divergierenden Ergebnissen, sodass der Effekt auf die adhäsive Grenzfläche zunächst weiterführender Untersuchungen bedarf (Amaral et al. 2007).

Während die pH-Wechselbelastung schon seit den 1980er Jahren in der Forschung über Kariesentstehung eingesetzt wird, ist zu ihrem Einfluss auf die Haftung von Kompositen bislang nur wenig bekannt. Die Wechselbelastungen scheinen die Effekte der durch Wasser ausgelösten Hydrolyse zu steigern (Sauro et al. 2006).

Als Nahrungsmittel-simulierende Lösungen werden meist Ethanol-Lösungen in verschiedenen Konzentrationen angewendet (Lee et al. 1994; Lee et al. 1996). Höher konzentrierte Ethanol-Lösungen scheinen die Kompositmatrix zu schwächen (Bagheri et al. 2005) und führen möglicherweise zu einer Kollagendegradation im Dentin (Lee et al. 1996). 


\subsubsection{Scherfestigkeits- und Bruchstellenanalyse}

Für Laboruntersuchungen zur Haftfestigkeit von Adhäsivsystemen bzw. Kompositen werden in der Zahnmedizin unter anderem Scherfestigkeits-, Zugfestigkeits- oder Bruchzähigkeitstests durchgeführt. Am häufigsten wird dabei die in der vorliegenden Studie durchgeführte Scherfestigkeitsanalyse mit einer nachfolgenden Bruchstellenanalyse angewendet (Braga et al. 2010; Heintze 2013). Scherfestigkeitsanalysen dentaler Materialien werden teilweise kritisch diskutiert, da die in der Literatur zu findenden Versuchsaufbauten nur wenig standardisiert sind und ihre Ergebnisse somit oft nicht direkt verglichen werden können (Al-Salehi und Burke 1997; Burke et al. 2008). Viele Faktoren, wie z. B. die Ausdehnung der Haftfläche, die Geschwindigkeit des Scherstempels der Testmaschine und die mögliche Durchführung eines artifiziellen Alterungsverfahrens variieren stark und nehmen dabei einen großen Einfluss auf die Ergebnisse (Leloup et al. 2001).

Dennoch lässt sich eine Korrelation der in vitro generierten Ergebnisse auf die klinische Anwendung der Materialien feststellen (Van Meerbeek et al. 2003; De Munck et al. 2005; Peumans et al. 2005). Es wird davon ausgegangen, dass eine Restauration umso länger in vivo überlebt, desto bessere Haftwerte des betreffenden Materials in vitro ermittelt werden konnten (Van Meerbeek et al. 2003).

Der Versuchsaufbau der vorliegenden Studie folgt den Empfehlungen verschiedener Autoren (Braga et al. 2010; Wiegand et al. 2012; Wiegand et al. 2015) und ist so gewählt, dass eine Einordnung in bereits bestehende Literatur möglich ist.

Der Einteilung in kohäsive, adhäsive und gemischte Versagensmuster liegt die Annahme zu Grunde, dass bei Vorliegen eines kohäsiven Bruchtyps ein stärkerer Verbund zwischen fließfähigem Komposit und Prüfkörperbasis vorliegt als bei adhäsiven Bruchmustern, da die adhäsive Interaktionsfläche beider Materialien intakt bleibt. 


\subsection{Ergebnisse}

In der vorliegenden Studie wiesen die selbstadhäsiven fließfähigen Komposite auf Schmelz ( $<5 \mathrm{MPa}$ ) und Dentin ( $<3 \mathrm{MPa}$ ) signifikant geringere Haftwerte als das Kontrollkomposit Venus Diamond Flow (Schmelz: 13,0 \pm 5,1 MPa; Dentin: 11,2 \pm 6,3 $\mathrm{MPa}$ ) und lediglich adhäsive und gemischte Versagensmuster auf. Auf NanoHybridkomposit erreichten die selbstadhäsiven Komposite bessere Haftwerte als auf Schmelz und Dentin. Die Reparaturhaftwerte waren signifikant abhängig von der Art der Vorbehandlung. Die Applikation des Adhäsivsystems verbesserte die Haftung des konventionellen, aber nicht der selbstadhäsiven Komposite signifikant. Insgesamt waren die Scherfestigkeiten von Venus Diamond Flow auf dem Nano-Hybridkomposit besser als die der selbstadhäsiven Komposite. Auf Nano-Hybridkomposit zeigten die selbstadhäsiven Komposite weniger adhäsive Versagensmuster, wenn eine mechanische Oberflächenbearbeitung durchgeführt wurde.

Die selbstadhäsiven fließfähigen Komposite unterscheiden sich hinsichtlich ihrer Zusammensetzung und besonders in der Art der verwendeten funktionellen Monomere. Fusio Liquid Dentin soll mittels der Carboxylatgruppen von 4Methacryloxyethyltrimelliticsäure (4-META) chemisch an das Hydroxylapatit der Zahnhartsubstanz binden (Yoshida et al. 2004). Das funktionelle Monomer Glycerophosphat-Dimethacrylat (GPDM) aus Vertise Flow wird auch im Goldstandard Etch\&Rinse-Adhäsivsystem Optibond FL eingesetzt. Durch Phosphatgruppen soll GPDM die Zahnoberfläche anätzen und an Kalzium binden (Kerr Dental Corporation 2010; Poitevin et al. 2013). Constic enthält funktionelle 10-Methacryloyloxydecyldihydrogenphosphat(MDP)-Monomere, die laut Herstellerangaben sowohl chemisch an Zahnhartsubstanz binden als auch eine Ätzwirkung auf diese ausüben sollen. MDP wird bereits seit einigen Jahren erfolgreich in selbstadhäsiven Kompositzementen eingesetzt (Ferracane et al. 2011).

Auch im Hinblick auf ihre Interaktion mit Zahnhartsubstanzen konnten Unterschiede zwischen den einzelnen selbstadhäsiven Kompositen festgestellt werden (Brueckner et al. 2017). Während nach Applikation eines experimentellen selbstadhäsiven fließfähigen Komposits (DMG America, selber Hersteller wie von dem in der vorliegenden Studie verwendeten Constic) oder von Vertise Flow im Schmelz deutliche Micro-Tags beobachtet werden konnten, bildete Fusio Liquid 
Dentin diese nur sehr unregelmäßig aus. Im Dentin wurden von allen drei selbstadhäsiven Kompositen Micro-Tags geformt, wobei diese von Vertise Flow und Fusio Liquid Dentin nur sehr dünn ausgebildet wurden, wohingegen das experimentelle System sogar verzweigte Tags bildete.

Eine weitere Studie zeigte in transmissionselektronenmikroskopischen Aufnahmen eine nur oberflächliche Interaktion von selbstadhäsiven fließfähigen Kompositen mit Zahnhartsubstanzen. Im Zahnschmelz entstanden nur sehr feine und kurze Micro-Tags und im Dentin bildete sich lediglich eine sehr dünne Hybridschicht aus (Hanabusa et al. 2011).

In verschiedenen In-vitro-Untersuchungen konnte gezeigt werden, dass die Haftung der selbstadhäsiven fließfähigen Komposite auf Schmelz und Dentin sowohl unmittelbar nach ihrer Applikation (Fu et al. 2013; Poitevin et al. 2013; Vichi et al. 2013; Makishi et al. 2015; DMG America) als auch nach artifizieller Alterung (Goracci et al. 2013; Veli et al. 2014; Brueckner et al. 2017) der Haftung von konventionellen Kompositen, die mit Adhäsivsystemen in der Etch\&Rinsebzw. Self-Etch-Technik angewendet wurden, unterlegen ist. Die in der vorliegenden Studie ermittelten Weibull-Module zeigen eine weit weniger zuverlässige Haftung der selbstadhäsiven Komposite $(m \leq 0,8)$ auf Schmelz und Dentin als das Kontrollkomposit ( $m \leq 2,8)$.

Die Interaktion der selbstadhäsiven Komposite mit Zahnhartsubstanzen wird besonders durch das Vorhandensein aprismatischen Schmelzes oder einer Schmierschicht auf Dentin weiter herabgesetzt (Mine et al. 2017).

Die Schmelzhaftung lässt sich durch vorherige selektive Schmelzätzung mit Phosphorsäure verbessern (Juloski et al. 2012; Poitevin et al. 2013), allerdings wird dadurch der Vorteil der Ein-Schritt-Technik eingebüßt.

Sowohl in der vorliegenden Studie, als auch in der Untersuchung von Brueckner et al. (2017) wurden nach Thermocycling keine signifikanten Unterschiede bezüglich der Haftwerte der selbstadhäsiven Komposite untereinander und im Vergleich zwischen Schmelz und Dentin festgestellt, obwohl diese doch sehr unterschiedlich mit den Zahnhartsubstanzen interagieren. Möglicherweise werden initial bestehende Unterschiede durch den Alterungseffekt des Thermolastwechsels aufgehoben.

Fließfähige Materialien weisen aufgrund des höheren Matrixanteils insgesamt eine vermehrte Wasseraufnahme als Komposite mit höherem Fülleranteil auf. Die 
hydrophilen Monomere der selbstätzenden und selbstadhäsiven Materialien zeigen gegenüber konventionellen fließfähigen Kompositen nochmals eine vermehrte Wasseraufnahme, dadurch kommt es zur Ausdehnung der Kompositmatrix und der Auflösung von Polymerketten (Wei et al. 2011b). So verschlechtern sich die mechanischen Eigenschaften der Komposite, es kommt zu einem signifikanten Abfall der Haftwerte nach Thermocycling (Brueckner et al. 2017). Schon nach 24 Stunden Lagerung in Wasser beobachteten Wei et al. sichtbare Risse in Prüfkörpern aus Vertise Flow. Sie schlussfolgerten, dass in der Matrix von Vertise Flow nur eine wenig ausgeprägte Verbindung zwischen den Monomeren und den Füllkörpern besteht, was in einem gesteigerten Verlust von Füllkörpern und einer erhöhten Degradation in Wasser resultiert (Wei et al. 2011a). Die schlechten In-vitro-Ergebnisse der selbstadhäsiven Komposite spiegeln sich auch in einer klinischen Untersuchung wider, in der bereits nach 6 Monaten $66 \%$ der mit selbstadhäsiven Kompositen angefertigten Klasse-VRestaurationen klinisch unakzeptabel waren bzw. einen Retentionsverlust aufwiesen (Çelik et al. 2015). Die klinische Anwendung selbstadhäsiver Komposite scheint somit derzeit wenig erfolgversprechend.

Während die Haftwerte der selbstadhäsiven Komposite auf Schmelz und Dentin sehr niedrig waren, konnten in Abhängigkeit von der Vorbehandlung deutlich bessere Reparaturhaftwerte und ein vermehrtes Auftreten von kohäsiven Bruchmustern auf Nano-Hybridkomposit gemessen werden. Die Weibull-Module der selbstadhäsiven Komposite als Reparaturmaterialien $(1,7 \leq m \geq 5,9)$ waren höher als auf Schmelz und Dentin ( $m \leq 0,8)$, die Reparaturhaftwerte können demnach als zuverlässiger angesehen werden als die Haftwerte auf Zahnhartsubstanz. Venus Diamond Flow erreichte als konventionelles Reparaturmaterial mit einem hohen Weibull-Modul von $m=9,7$ die zuverlässigsten Haftfestigkeiten von bis zu 24,4 MPa. In Übereinstimmung zu früheren Untersuchungen zur Verwendung konventioneller Komposite als Reparaturmaterialien (Loomans et al. 2011; Hickel et al. 2013) führte die mechanische Konditionierung der Reparaturoberfläche sowie die Benetzung dieser mit einem Adhäsivsystem insgesamt zu besseren Reparaturhaftwerten der Kontrollgruppe als der Verzicht auf eine entsprechende Konditionierung. Im Gegensatz dazu wurden die Haftwerte der selbstadhäsiven Komposite auf Nano-Hybridkomposit 
zwar ebenfalls von der Art der mechanischen Oberflächenkonditionierung der Reparaturfläche, allerdings nicht von der zusätzlichen Verwendung von Optibond FL beeinflusst. Möglicherweise sind die selbstadhäsiven fließfähigen Komposite weniger viskös als das Kontrollkomposit und können die durch die unterschiedlichen Verfahren angerauten Kompositoberflächen besser benetzen als Venus Diamond Flow, welches daher bessere Haftwerte aufweist, wenn zuvor noch ein niedrigvisköses Adhäsiv aufgetragen wird.

Insgesamt erreichte nur das Material Fusio Liquid Dentin Reparaturhaftwerte, die mit denen des konventionellen fließfähigen Komposits vergleichbar sind. Schon während der Anwendung konnte sowohl in der vorliegenden Studie als auch in einer weiteren (Poitevin et al. 2013) bei Fusio Liquid Dentin eine niedrigere Viskosität und daraus resultierend eine bessere Benetzbarkeit im Vergleich zu den anderen selbstadhäsiven fließfähigen Kompositen festgestellt werden.

Die Ergebnisse der Scherfestigkeitsanalyse wurden durch eine Weibull-Analyse vervollständigt, die Aussagen über die Streuung der Ergebnisse ermöglicht. Dabei spiegelt sich eine große Streuung der Scherfestigkeiten in einem niedrigen Weibull-Modul wider, woraus sich eine erniedrigte Zuverlässigkeit der charakteristischen Festigkeit ergibt. Im Allgemeinen sollten Materialien mit einem höheren Weibull-Modul denen mit niedrigeren Werten bevorzugt werden, da diese meist weniger techniksensibel sind (Scherrer et al. 2010). In der vorliegenden Studie erreichte das Kontrollkomposit Venus Diamond Flow sowohl auf Zahnhartsubstanz als auch auf Nano-Hybridkomposit die höchsten WeibullModule der getesteten fließfähigen Komposite, sein Einsatz kann also dem der selbstadhäsiven Komposite bevorzugt werden.

Die Weibull-Module der selbstadhäsiven Komposite waren auf Schmelz und Dentin weitaus geringer als auf Nano-Hybridkomposit, die ermittelten Reparaturhaftwerte können demnach als zuverlässiger angesehen werden als die Haftwerte auf Zahnhartsubstanz.

Die Nullhypothese, dass die Haftwerte der selbstadhäsiven fließfähigen Komposite sich nicht signifikant von einem konventionellen fließfähigen Komposit unterscheiden, wurde den Ergebnissen dieser Studie folgend abgelehnt. 


\subsection{Schlussfolgerungen}

Innerhalb der Limitationen der vorliegenden Studie zeigen selbstadhäsive fließfähige Komposite im Vergleich zu einem konventionellen Komposit in Kombination mit einem Etch\&Rinse-Adhäsivsystem auf Schmelz und Dentin schlechte Haftfestigkeiten. Auch unter Berücksichtigung weiterer In-vitro-Studien (Fu et al. 2013; Goracci et al. 2013; Poitevin et al. 2013; Vichi et al. 2013; Veli et al. 2014; Makishi et al. 2015; Brueckner et al. 2017; DMG America) kann die Verwendung dieser Materialien für die direkte Füllungstherapie derzeit nicht empfohlen werden.

Auf Nano-Hybridkomposit zeigen die selbstadhäsiven Komposite jedoch bessere Haftwerte als auf Zahnhartsubstanz. Daher sind sie möglicherweise für die Reparatur defekter Kompositrestaurationen geeignet, sofern es sich um kleinere, rein kompositbegrenzte Reparaturareale handelt. 


\section{Zusammenfassung}

Hintergrund und Ziel der Untersuchung: Selbstadhäsive Komposite für die direkte Füllungstherapie sollen ohne vorherige Konditionierung der Zahnhartsubstanz mit einem Adhäsivsystem angewendet werden können und stellen somit eine Vereinfachung der Adhäsivtechnik dar. Bislang ist allerdings kaum untersucht, ob die klinische Erfolgsrate dieser Restaurationen mit konventionellen Kompositen vergleichbar ist. Ferner ist nicht bekannt, ob selbstadhäsive Komposite auch als Reparaturmaterialien zur Behandlung partiell defekter dentaler Restaurationen geeignet sind. Das Ziel dieser Studie war es daher, die Scherfestigkeit von drei selbstadhäsiven fließfähigen Kompositen auf Schmelz, Dentin und einem NanoHybridkomposit im Vergleich zu einem konventionellen fließfähigen Komposit nach Alterung zu untersuchen.

Material und Methoden: Die selbstadhäsiven Komposite (Constic, DMG America; Fusio Liquid Dentin, Pentron Clinical; Vertise Flow, Kerr Dental) wurden nach Herstellerangabe auf Schmelz und Dentin appliziert; das konventionelle Komposit (Venus Diamond Flow, Heraeus Kulzer) wurde mit einem Adhäsivsystem in Etch\&Rinse-Technik (Optibond FL, Kerr Dental) angewendet. Die NanoHybridkompositprüfkörper (Venus Diamond, Heraeus Kulzer) wurden zunächst einem Thermolastwechsel (5000 Zyklen, 5-55 ${ }^{\circ} \mathrm{C}$ ) unterzogen. Um eine Reparaturrestauration zu simulieren, wurden die Prüfkörperoberflächen mechanisch angeraut, mit Aluminiumoxid korundgestrahlt, silikatisiert/silanisiert oder nicht vorbehandelt. Die Hälfte der Prüfkörper wurde dann mit einem Adhäsivsystem (Optibond FL, Kerr Dental) behandelt, bevor eines der fließfähigen Komposite appliziert wurde. Alle Prüfkörper (je Untergruppe $n=16$ ) wurden einem Thermolastwechsel (5000 Zyklen, $5-55^{\circ} \mathrm{C}$ ) unterzogen, bevor die Scherfestigkeit gemessen und die Versagensmuster analysiert wurden. Die statistische Auswertung erfolgte mittels ANOVAs und Bonferroni-post-hoc-Tests $(p<0,05)$, außerdem wurde eine Weibull-Statistik durchgeführt.

Ergebnisse: Die Scherfestigkeiten der selbstadhäsiven Komposite auf Schmelz $(<5$ $\mathrm{MPa}$ ) und Dentin ( $<3 \mathrm{MPa}$ ) waren signifikant geringer als die von Venus Diamond Flow (Schmelz: 13,0 $\pm 5,1 \mathrm{MPa}$; Dentin: 11,2 $\pm 6,3 \mathrm{MPa}$ ). Die Haftung der Materialien auf dem Nano-Hybridkomposit war signifikant abhängig von der Art der Vorbehandlung. Die Applikation des Adhäsivsystems verbesserte die Scherfestigkeit 
des konventionellen, aber nicht der selbstadhäsiven Komposite signifikant. Die Anwendung der selbstadhäsiven fließfähigen Komposite führte zumeist zu adhäsiven Versagensmustern, die allerdings durch eine mechanische Oberflächenbehandlung reduziert werden konnten.

Schlussfolgerung: Zusammenfassend kann festgestellt werden, dass selbstadhäsive fließfähige Komposite auf Schmelz und Dentin schlechte Haftfestigkeiten zeigen und somit nicht für die direkte Füllungstherapie empfohlen werden können, möglicherweise aber zur Reparatur defekter Kompositrestaurationen geeignet sind. 


\section{Literaturverzeichnis}

Al-Salehi S, Burke F (1997): Methods used in dentin bonding tests: an analysis of 50 investigations on bond strength. Quintessence Int $\underline{28}, 717-23$

Amaral FLB, Colucci V, Palma-Dibb RG, Corona SAM (2007): Assessment of in vitro methods used to promote adhesive interface degradation: A critical review. J Esthet Restor Dent 19, 340-353

Bagheri R, Burrow MF, Tyas M (2005): Influence of food-simulating solutions and surface finish on susceptibility to staining of aesthetic restorative materials. J Dent $\underline{33}$, 389-398

Baroudi K, Rodrigues JC (2015): Flowable resin composites: A systematic review and clinical considerations. J Clin Diagn Res $\underline{9}$, ZE18-24

Belli R, Pelka M, Petschelt A, Lohbauer U (2009): In vitro wear gap formation of selfadhesive resin cements: a CLSM evaluation. J Dent 37, 984-993

Braga RR, Meira JBC, Boaro LCC, Xavier TA (2010): Adhesion to tooth structure: A critical review of "macro“ test methods. Dent Mater $\underline{26}$, e38-49

Brendeke J, Özcan M (2007): Effect of physicochemical aging conditions on the composite-composite repair bond strength. J Adhes Dent $\underline{9}$, 399-406

Brueckner C, Schneider H, Haak R (2017): Shear bond strength and tooth-composite interaction with self-adhering flowable composites. Oper Dent $\underline{42}, 90-100$

Burke F, Hussain A, Nolan L, Fleming G (2008): Methods used in dentine bonding tests: an analysis of 102 investigations on bond strength. Eur J Prosthodont Restor Dent $\underline{16}, 158-65$

Carrilho MRO, Carvalho RM, Tay FR, Pashley DH (2004): Effects of storage media on mechanical properties of adhesive systems. Am J Dent 17, 104-108

Çelik EU, Aka B, Yilmaz F (2015): Six-month clinical evaluation of a self-adhesive flowable composite in noncarious cervical lesions. J Adhes Dent 17, 361-8

Cramer N, Stansbury J, Bowman C (2011): Recent advances and developments in composite dental restorative materials. J Dent Res $\underline{90}$, 402-416

De Munck J, Van Landuyt K, Peumans M, Poitevin A, Lambrechts P, Braem M, Van Meerbeek B (2005): A critical review of the durability of adhesion to tooth tissue: methods and results. J Dent Res $\underline{84}, 118-132$

DMG America: Constic. The New 3-in-1 Flowable: Etching, Bonding and Filling in One Step! [http://www.dmg-america.com/files/product/literature/[nid]/Constic_BrochureDMGA_FINAL.pdf; Zugriff am 28.01.2017]

Fernández E, Martín J, Vildósola P, Oliveira OB, Gordan V, Mjor I, Bersezio C, Estay J, De Andrade MF, Moncada G (2015): Can repair increase the longevity of composite resins? Results of a 10-year clinical trial. J Dent $\underline{43}, 279-86$

Ferracane JL (2008): Placing dental composites_A stressful experience. Oper Dent $\underline{33}$, 247-257 
Ferracane JL (2011): Resin composite-State of the art. Dent Mater 27, 29-38

Ferracane JL, Stansbury JW, Burke FJT (2011): Self-adhesive resin cements - chemistry, properties and clinical considerations. J Oral Rehabil 38, 295-314

Frankenberger R, Tay $F$ (2005): Self-etch vs etch-and-rinse adhesives: effect of thermomechanical fatigue loading on marginal quality of bonded resin composite restorations. Dent Mater 21, 397-412

Frankenberger R, Krämer N, Petschelt A (2000): Technique sensitivity of dentin bonding: effect of application mistakes on bond strength and marginal adaptation. Oper Dent 25, 324-330

Frankenberger R, Krämer N, Lohbauer U, Nikolaenko S, Reich S (2007): Marginal integrity: is the clinical performance of bonded restorations predictable in vitro? J Adhes Dent $\underline{9}, 107-16$

Fu J, Kakuda S, Pan F, Hoshika S, Ting S, Fukuoka A, Bao Y, Ikeda T, Nakaoki Y, Selimovic D, et al. (2013): Bonding performance of a newly developed step-less all-in-one system on dentin. Dent Mater J $\underline{32}$, 203-11

Gale M, Darvell B (1999): Thermal cycling procedures for laboratory testing of dental restorations. J Dent $\underline{27}, 89-99$

Garcia-Godoy F, Krämer N, Feilzer AJ, Frankenberger R (2010): Long term degradation of enamel and dentin bonds: 6 -year results in vitro vs. in vivo. Dent Mater $\underline{26}$, $1113-1118$

Goracci C, Margvelashvili M, Giovannetti A, Vichi A, Ferrari M (2013): Shear bond strength of orthodontic brackets bonded with a new self-adhering flowable resin composite. Clin Oral Investig 17, 609-617

Gwinnett A (1981): Acid etching for composite resins. Dent Clin North Am 25, 271-289

Hanabusa M, Mine A, Kuboki T, Momoi Y, Van Landuyt KL, Van Meerbeek B, De Munck J (2011): TEM interfacial characterization of an experimental self-adhesive filling material bonded to enamel/dentin. Dent Mater $\underline{27}, 818-24$

Hannig C, Laubach S, Hahn P, Attin T (2006): Shear bond strength of repaired adhesive filling materials using different repair procedures. J Adhes Dent $\underline{8}, 35-40$

Hashimoto M, Ohno H, Sano H, Kaga M, Oguchi H (2003): In vitro degradation of resindentin bonds analyzed by microtensile bond test, scanning and transmission electron microscopy. Biomaterials $\underline{24}, 3795-3803$

Hebling J, Castro F, Costa C (2007): Adhesive performance of dentin bonding agents applied in vivo and in vitro. Effect of intrapulpal pressure and dentin depth. J Biomed Mater Res B Appl Biomater 83, 295-303

Heintze SD (2013): Clinical relevance of tests on bond strength, microleakage and marginal adaptation. Dent Mater $\underline{29}, 59-84$

Hickel R, Heidemann D, Staehle H, Minnig P, Wilson M (2004): Direct composite restorations: extended use in anterior and posterior situations. Clin Oral Investig $\underline{8}$, $43-44$ 
Hickel R, Brüshaver K, llie N (2013): Repair of restorations - Criteria for decision making and clinical recommendations. Dent Mater 29, 28-50

Ilie N, Hickel R (2011): Resin composite restorative materials. Aust Dent J $\underline{56}, 59-66$

Imbery T, Gray T, DeLatour F, Boxx C, Best A, Moon P (2014): Evaluation of Flexural, Diametral Tensile, and Shear Bond Strength of Composite Repairs. Oper Dent 39, E250-E260

International Organization for Standardization: ISO TS11405 Dental materials: testing of adhesion to tooth structure. Technical specification. 2003 [https://www.iso.org/standard/62898.html; Zugriff am 03.12.2014]

Janda R (1988): Der Stand der Entwicklung auf dem Gebiet der Zahnfüllungskunststoffe (I-III). Quintessenz 39, 1067-73, 1243-53, 1393-8

Juloski J, Goracci C, Rengo C, Giovannetti A, Vichi A, Vulicevic Z, Ferrari M (2012): Enamel and dentin bond strength of new simplified adhesive materials with and without preliminary phosphoric acid-etching. Am J Dent 25, 239-43

Kanzow P, Hoffmann R, Tschammler C, Kruppa J, Rödig T, Wiegand A (2016a): Attitudes, practice, and experience of German dentists regarding repair restorations. Clin Oral Investig $\underline{21}, 1087-193$

Kanzow P, Wiegand A, Schwendicke F (2016b): Cost-effectiveness of repairing versus replacing composite or amalgam restorations. J Dent Nov, 41-47

Kerr Dental Corporation (2010): Vertise Flow Technical bulletin. [ https:// www.kerrdental.com/resourcecenter?search=technical+bulletin+vertise+flow; Zugriff am: 28.12.2016]

Lee SY, Greener EH, Mueller HJ, Chiu CH (1994): Effect of food and oral simulating fluids on dentine bond and composite strength. J Dent 22, 352-359

Lee SY, Greener EH, Covey DA, Menis DL (1996): Effects of food/oral simulating fluids on microstructure and strength of dentine bonding agents. J Oral Rehabil $\underline{23}, 353-$ 361

Leloup G, D'Hoore W, Bouter D, Degrange M, Vreven J (2001): Meta-analytical review of factors involved in dentin adherence. J Dent Res $\underline{80}, 1605-14$

Loomans B, Vivan Cardoso M, Roeters F, Opdam N, De Munck J, Huysmans MCDNJM, Van Meerbeek B (2011): Is there one optimal repair technique for all composites? Dent Mater 27, 701-709

Lutz F, Phillips R (1983): A classification and evaluation of composite resin systems. J Prothet Dent $\underline{50}$, 480-488

Magne P, Mahallati R, Bazos P, So W (2008): Direct dentin bonding technique sensitivity when using air/suction drying steps. J Esthet Restor Dent 130-8

Makishi P, Pacheco RR, Sadr A, Shimada Y, Sumi Y, Tagami J, Giannini M (2015): Assessment of self-adhesive resin composites: Nondestructive imaging of resindentin interfacial adaptation and shear bond strength. Microsc Microanal $\underline{21}$, $1523-1529$ 
Maneenut C, Sakoolnamarka R, Tyas M (2011): The repair potential of resin composite materials. Dent Mater $\underline{27}, 20-7$

Manhart J, Chen HY, Hickel R (2001): The suitability of packable resin-based composites for posterior restorations. J Am Dent Assoc 1939 132, 639-645

Manhart J, Chen HY, Hamm G, Hickel R (2004): Buonocore Memorial Lecture. Review of the clinical survival of direct and indirect restorations in posterior teeth of the permanent dentition. Oper Dent 29, 481-508

Mehl A, Kunzelmann K-H, Folwaczny M, Hickel R (2004): Stabilization effects of CAD/CAM ceramic restorations in extended MOD cavities. J Adhes Dent $\underline{6}, 239$ 245

Mine A, De Munck J, Van Ende A, Poitevin A, Matsumoto M, Yoshida Y, Kuboki T, Van Landuyt KL, Yatani H, Van Meerbeek B (2017): Limited interaction of a selfadhesive flowable composite with dentin/enamel characterized by TEM. Dent Mater $\underline{33}, 209-217$

Mjør I, Fejerskov O: Human Oral embryology and histology. Munksgaard, Kopenhagen 1986

Moncada G, Martin J, Fernandez E, Hempel M (2009): Sealing, refurbishment and repair of Class I and Class II defective restorations: a three-year clinical trial. Am Dent Assoc 140, 425-32

Oliveira S, Pugach M, Hilton J, Watanabe L, Marshall S, Marshall GJ (2003): The influence of the dentin smear layer on adhesion: a self-etching primer vs. a totaletch system. Dent Mater $\underline{19}, 758-767$

Özcan M, Raadschelders J, Vallittu P, Lassilla L (2013): Effect of particle deposition parameters on silica coating of zirconia using a chairside air-abrasion device. $\mathrm{J}$ Adhes Dent 15, 211-4

Ozer F, Blatz MB (2013): Self-etch and etch-and-rinse adhesive systems in clinical dentistry. Compend Contin Educ Dent 34, 12-14, 16, 18; quiz 20,30

Patel B, Chhabra N, Jain D (2016): Effect of different polishing systems on the surface roughness of nano-hybrid composites. J Conserv Dent $\underline{19}, 37-40$

Perdigao J, Frankenberger R (2001): Effect of solvent and rewetting time on dentin adhesion. Quintessence Int $\underline{32}, 385-390$

Perdigao J, Lambrechts P, Van Meerbeek B, Braem M, Yildiz E, Yucel T (1996): The interaction of adhesive systems with human dentin. Am J Dent $\underline{9}, 167-173$

Perdigao J, Frankenberger R, Rosa B, Breschi L (2000): New trends in dentin/enamel adhesion. Am J Dent 13, 25D-30D

Peumans M, Kanumilli P, De Munck J, Van Landuyt K, Lambrechts P, Van Meerbeek B (2005): Clinical effectiveness of contemporary adhesives: a systematic review of current clinical trials. Dent Mater 21, 864-81

Peumans M, De Munck J, Van Landuyt KL, Poitevin A, Lambrechts P, Van Meerbeek B (2012): A 13-year clinical evaluation of two three-step etch-and-rinse adhesives in non-carious class-V lesions. Clin Oral Investig $\underline{16}, 129-137$ 
Peutzfeldt A (1997): Resin composites in dentistry: the monomer systems. Eur J Oral Sci $\underline{105}, 97-116$

Poitevin A, De Munck J, Van Ende A, Suyama Y, Mine A, Peumans M, Van Meerbeek B (2013): Bonding effectiveness of self-adhesive composites to dentin and enamel. Dent Mater 29, 221-230

Rinastiti M, Özcan M, Siswomihardjo W, Busscher HJ (2011): Effects of surface conditioning on repair bond strengths of non-aged and aged microhybrid, nanohybrid, and nanofilled composite resins. Clin Oral Investig 15, 625-633

Rodrigues Jr. SA, Ferracane JL, Bona ÁD (2009): Influence of surface treatments on the bond strength of repaired resin composite restorative materials. Dent Mater $\underline{25}$, 442-451

Sauro S, Watson TF, Tay FR, Chersoni S, Breschi L, Bernardi F, Prati C (2006): Water uptake of bonding systems applied on root dentin surfaces: a SEM and confocal microscopic study. Dent Mater $\underline{22}, 671-680$

Scherrer SS, Cesar PF, Swain MV (2010): Direct comparison of the bond strength results of the different test methods: A critical literature review. Dent Mater $\underline{26}$, e78-93

Spreafico D, Semeraro S, Mezzanzanica D, Re D, Gagliani M, Tanaka T (2006): The effect of the air-blowing step on the technique sensitivity of four different adhesive systems. J Dent 3, 237-44

Van Meerbeek B, Lambrechts P, Inokoshi S, Braem M, Vanherle G (1992): Factors affecting adhesion to mineralized tissues. Oper Dent $\underline{5}, 111-124$

Van Meerbeek B, De Munck J, Yoshida Y, Inoue S, Vargas M, Vijay P, Van Landuyt K, Lambrechts P, Vanherle G (2003): Buonocore Memorial Lecture Adhesion to enamel and dentin: Current status and future challenges. Oper Dent 28, 215-235

Van Meerbeek B, Van Landuyt K, De Munck J, Hashimoto M, Peumans M, Lambrechts P (2005): Technique-sensitivity of contemporary adhesives. Dent Mater J 1-13

Veli I, Akin M, Kucukyilmaz E, Uysal T (2014): Shear bond strength of a self-adhering flowable composite when used for lingual retainer bonding. J Orofac Orthop $\underline{75}$, 374-383

Vichi A, Margvelashvili M, Goracci C, Papacchini F, Ferrari M (2013): Bonding and sealing ability of a new self-adhering flowable composite resin in class I restorations. Clin Oral Investig 17, 1497-506

Wei YJ, Silikas N, Zhang ZT, Watts DC (2011a): Diffusion and concurrent solubility of selfadhering and new-resin-matrix composites during water sorption/desorption cycles. Dent Mater 27, 197-205

Wei YJ, Silikas N, Zhang ZT, Watts DC (2011b): Hygroscopic dimensional changes of self-adhering and new resin-matrix composites during water sorption/desorption cycles. Dent Mater 27, 259-66

Wei YJ, Silikas N, Zhang ZT, Watts DC (2013): The relationship between cyclic hygroscopic dimensional changes and water sorption/desorption of self-adhering and new resin-matrix composites. Dent Mater $\underline{29}$, e218-e226 
Wiegand A, Stawarczyk B, Buchalla W, Tauböck TT, Özcan M, Attin T (2012): Repair of silorane composite-Using the same substrate or a methacrylate-based composite? Dent Mater 28, e19-e25

Wiegand A, Stucki L, Hoffmann R, Attin T, Stawarczyk B (2015): Repairability of CAD/CAM high-density PMMA- and composite-based polymers. Clin Oral Investig $\underline{19}, 2007-2013$

Yoshida Y, Nagakane K, Fukuda R, Nakayama Y, Okazaki M, Shintani H, Inoue S, Tagawa Y, Suzuki K, De Munck J, Van Meerbeek B (2004): Comparative study on adhesive performance of functional monomers. J Dent Res $\underline{83}, 454-458$ 


\section{Danksagung}

Mein Dank gilt allen, die mich bei der Anfertigung dieser Dissertation unterstützt haben:

Frau Prof. Dr. Annette Wiegand danke ich für die Bereitstellung des interessanten Themas und die hervorragende Betreuung.

Frau Dr. Marta Rizk möchte ich für die besondere Unterstützung während des gesamten Entstehungsprozesses dieser Dissertation danken.

Weiterhin gilt mein Dank Frau Monika Hoch und den Mitarbeiterinnen und Mitarbeitern des Labors der Mund-Kiefer-Gesichtschirurgie für die Hilfestellungen während der Labortätigkeiten. 


\section{Lebenslauf}

Am 20.10.1989 wurde ich in Hannover-Langenhagen als Kind von Ursula und Hans-Heinrich Peterson geboren.

Von 1996 bis 2000 besuchte ich die Friedrich-Ebert-Grundschule in Hannover und von 2000 bis 2002 die Orientierungsstufe Badenstedt in Hannover.

Von 2002 bis 2009 war ich Schülerin des Gymnasiums Humboldtschule Hannover und erlangte dort 2009 die allgemeine Hochschulreife.

Zwischenzeitlich besuchte ich von 2006-2007 die Highschool in Royse City Texas, USA.

Das Studium der Zahnheilkunde begann ich 2009 an der Georg-AugustUniversität Göttingen und erhielt dort 2014 meine Approbation als Zahnärztin. Seit 2015 arbeite ich als Assistenzzahnärztin in der Poliklinik für Präventive Zahnmedizin, Parodontologie und Kariologie der Universitätsmedizin Göttingen. 